\title{
European multicenter study on antimicrobial resistance in bacteria isolated from companion animal urinary tract infections
}

Cátia Marques ${ }^{1}$, Luís Telo Gama ${ }^{1}$, Adriana Belas ${ }^{1}$, Karin Bergström² ${ }^{2}$ Stéphanie Beurlet ${ }^{3}$, Alexandra Briend-Marchal ${ }^{3}$, Els M. Broens ${ }^{4}$, Marta Costa ${ }^{5}$, Delphine Criel ${ }^{6}$, Peter Damborg ${ }^{7}$, Marloes A. M. van Dijk ${ }^{4}$, Astrid M. van Dongen ${ }^{8}$, Roswitha Dorsch ${ }^{9}$, Carmen Martin Espada ${ }^{10}$, Bernhard Gerber ${ }^{11}$, Maria Kritsepi-Konstantinou ${ }^{12}$, Igor Loncaric ${ }^{13}$, Domenico Mion ${ }^{14}$, Dusan Misic ${ }^{15}$, Rebeca Movilla ${ }^{16}$, Gudrun Overesch ${ }^{17}$, Vincent Perreten ${ }^{17}$, Xavier Roura ${ }^{16}$, Joachim Steenbergen ${ }^{6}$, Dorina Timofte ${ }^{18,19,20}$, Georg Wolf ${ }^{21}$, Renato Giulio Zanoni ${ }^{14}$, Sarah Schmitt ${ }^{22}$, Luca Guardabassi ${ }^{7,23}$ and Constança Pomba ${ }^{1 *}$ (D)

\begin{abstract}
Background: There is a growing concern regarding the increase of antimicrobial resistant bacteria in companion animals. Yet, there are no studies comparing the resistance levels of these organisms in European countries. The aim of this study was to investigate geographical and temporal trends of antimicrobial resistant bacteria causing urinary tract infection (UTI) in companion animals in Europe. The antimicrobial susceptibility of 22256 bacteria isolated from dogs and cats with UTI was determined. Samples were collected between 2008 and 2013 from 16 laboratories of 14 European countries. The prevalence of antimicrobial resistance of the most common bacteria was determined for each country individually in the years 2012-2013 and temporal trends of bacteria resistance were established by logistic regression.
\end{abstract}

Results: The aetiology of uropathogenic bacteria differed between dogs and cats. For all bacterial species, Southern countries generally presented higher levels of antimicrobial resistance compared to Northern countries. Multidrug-resistant Escherichia coli were found to be more prevalent in Southern countries. During the study period, the level of fluoroquinolone-resistant E. coli isolated in Belgium, Denmark, France and the Netherlands decreased significantly. A temporal increase in resistance to amoxicillin-clavulanate and gentamicin was observed among E. coli isolates from the Netherlands and Switzerland, respectively. Other country-specific temporal increases were observed for fluoroquinolone-resistant Proteus spp. isolated from companion animals from Belgium.

Conclusions: This work brings new insights into the current status of antimicrobial resistance in bacteria isolated from companion animals with UTI in Europe and reinforces the need for strategies aiming to reduce resistance.

Keywords: Antimicrobial resistance, Temporal trends, MRSA, MRSP, Dog, Cat

\footnotetext{
* Correspondence: cpomba@fmv.ulisboa.pt

${ }^{1}$ Faculdade de Medicina Veterinária, Centro de Investigação Interdisciplinar

em Sanidade Animal (CIISA), Lisboa, Portugal

Full list of author information is available at the end of the article
}

\section{Biomed Central}

(c) 2016 The Author(s). Open Access This article is distributed under the terms of the Creative Commons Attribution 4.0 International License (http://creativecommons.org/licenses/by/4.0/), which permits unrestricted use, distribution, and reproduction in any medium, provided you give appropriate credit to the original author(s) and the source, provide a link to the Creative Commons license, and indicate if changes were made. The Creative Commons Public Domain Dedication waiver (http://creativecommons.org/publicdomain/zero/1.0/) applies to the data made available in this article, unless otherwise stated. 


\section{Background}

Bacterial urinary tract infections (UTI) are frequently diagnosed in dogs and are considered rare in cats $[1,2]$. Lately, increased frequencies of UTI in cats have been reported in some European countries [3-5] in particularly when concurrent diseases are present [6].

Escherichia coli is the most frequent isolated bacteria causing UTI in dogs and cats. Several studies show frequencies greater than $30 \%$ [7-9]. Other commonly isolated bacteria genera include Staphylococcus spp., Enterococcus spp., Proteus spp. and Klebsiella spp. [7-10].

Previous studies in the United Kingdom and in Missouri-Columbia (USA) analysing the temporal trends of antimicrobial resistance in small collections of bacterial isolates from companion animal infections point to a significant increase in antimicrobial resistance [11, 12]. Furthermore, the emergence of multidrug-resistant bacteria (isolates resistant to three or more antimicrobial categories) in companion animals is an increasing concern [11, 13-15]. This creates new therapeutic challenges in veterinary medicine and is also a public health issue, since these pathogens may be zoonotic [16] and companion animals may play a role in the spread of resistant bacteria due to their close contact to humans [14, 17].

Antimicrobial resistance may vary according to the geographic location $[9,18]$. Data on antimicrobial resistance in bacteria isolated from companion animals with UTI in Europe are not easily comparable due to differences in study design, such as variations in host species, inclusion criteria and/or time period. Thus, it is difficult to get a European overview of antimicrobial resistance as seen in human medicine surveillance programmes such as the European Antimicrobial Resistance Surveillance Network [18].

Antimicrobial therapy in UTI should ideally rely on susceptibility testing of the isolated bacteria [19]. Yet, antimicrobials are frequently administered empirically based on the presence of compatible clinical signs, urine cytological findings and in the absence of urine culture and are required to alleviate UTI symptoms while waiting for antimicrobial susceptibility testing results [19]. Besides the pharmacokinetic-pharmacodynamic properties, the empiric antimicrobial selection should consider the most likely causative agent as well as its regional susceptibility patterns [8]. Moreover, according to the World Organisation for Animal Health [20], veterinarians should adopt strategies aimed at the reduction of antimicrobial resistance. Therefore, current information on the aetiology and antimicrobial resistance focused on UTI is of crucial importance.

Under the umbrella of the European Society of Veterinary Nephrology and Urology, a multicenter retrospective study was launched in November 2013 with the goal of getting antimicrobial resistance data on bacteria isolated from companion animal with UTI across Europe. A Urinary Tract Infection Resistance - Veterinary Network (UTIR-VNet) was constituted with this purpose in mind. Partial results were presented at the annual Society meeting included in the $25^{\text {th }}$ congress of the European College of Veterinary Internal Medicine, 4-6 September 2014, Mainz, Germany. The aim of this study was to determine the frequency of uropathogens in dogs and cats with urinary tract infection in Europe and to characterise the frequency and temporal trends of antimicrobial resistance over a period of six years. We hereby present a complete report and discussion of this study.

\section{Methods}

\section{Participating countries}

Between January and September 2014, 16 veterinary microbiology laboratories from 14 European countries (Austria, Belgium, Denmark, France, Germany, Greece, Italy, the Netherlands, Portugal, Serbia, Spain, Sweden, Switzerland, United Kingdom), were invited to participate in this study (Fig. 1). Laboratories were requested to send available retrospective data on animal species, age and gender, bacterial identification and antimicrobial susceptibility testing conducted in bacteria obtained from dogs and cats with UTI between 2008 and 2013. Samples were obtained with owners consent as part of the routine care of canine and feline UTI.

\section{Bacterial Isolates}

The bacteria identification varied between laboratories. Most laboratories used standard phenotypic tests, including API, while others used techniques such as PCR and MALDI-TOF. This discrepancy was particularly evident for staphylococci, which were classified to either the species or genus level depending on the laboratory.

\section{Susceptibility testing}

The following antimicrobials were included: amoxicillinclavulanate (AMC), ampicillin (AMP), cefotaxime (CTX), cefovecin $(\mathrm{CVN})$, cefoxitin (FOX), ceftazidime (CAZ), cefpodoxime (CPD), ceftiofur (EFT), ciprofloxacin (CIP), enrofloxacin (ENR), gentamicin (CN), marbofloxacin (MAR), oxacillin (OX), penicillin (P) and trimethoprim/ sulfamethoxazole (SXT).

The retrospective nature of the study forced us to include two in vitro antimicrobial susceptibility testing methods. Laboratories from Austria, France, Germany, Greece, Italy, the Netherlands, Portugal, Serbia, Spain, United Kingdom, used standard disc diffusion method according to Clinical Laboratory Standards Institute (CLSI) guidelines [21], whereas Sweden (VetMIC, SVA, Uppsala, Sweeden), Denmark and United Kingdom (COMPAN1F Sensititre panels, Thermo Fisher), Switzerland and 


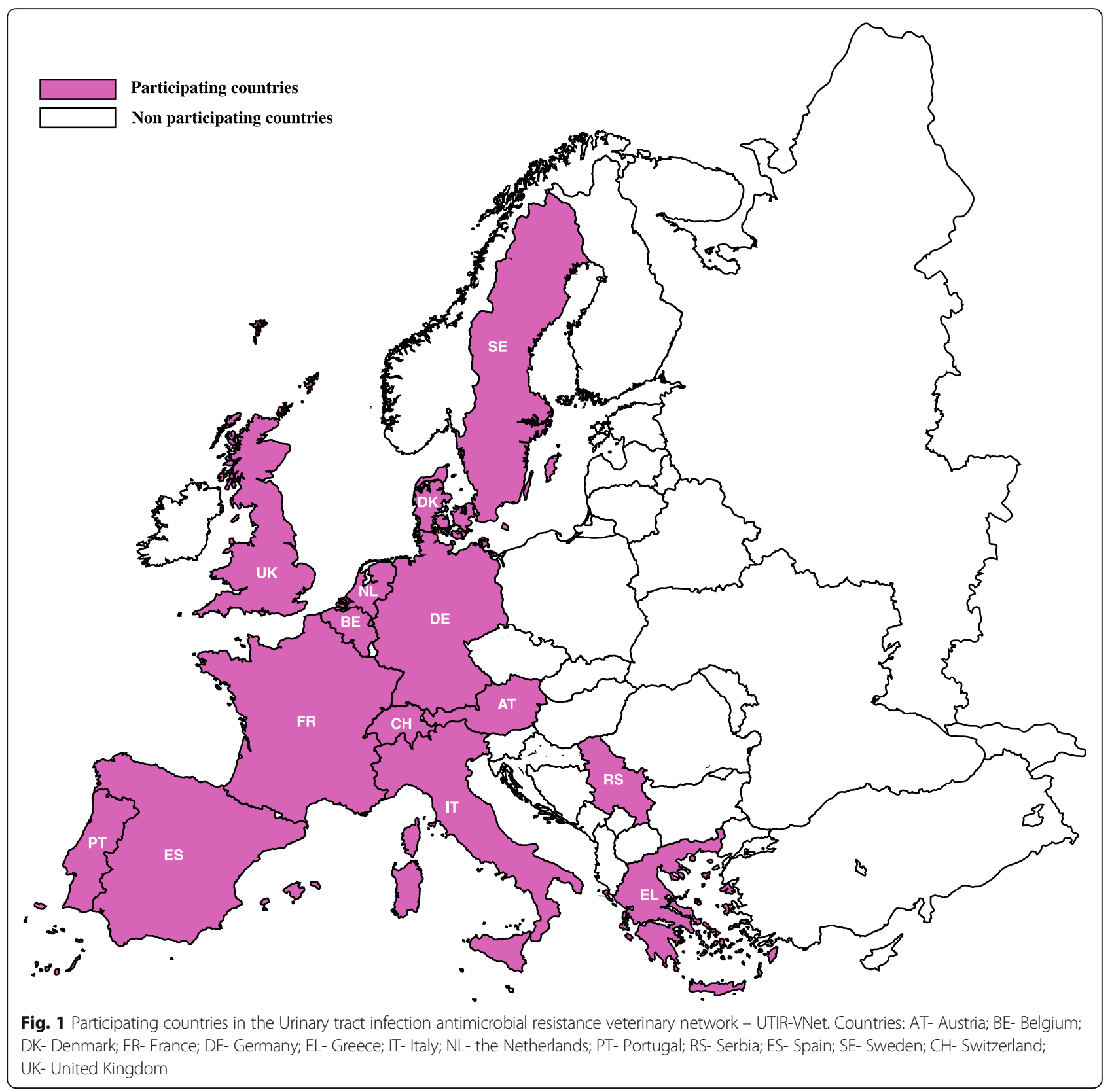

Belgium (VITEK 2, BioMérieux) used broth microdilution method.

Human CLSI breakpoints [22] were used for interpretation of minimal inhibitory concentration and disk diffusion results for CAZ (30 $\mu \mathrm{g})$, CTX $(30 \mu \mathrm{g})$, and CIP $(5 \mu \mathrm{g})$, whereas veterinary CLSI breakpoints [23] were used for AMC $(30 \mu \mathrm{g})$, AMP $(30 \mu \mathrm{g}), \mathrm{CN}(10 \mu \mathrm{g}), \mathrm{CPD}(10 \mu \mathrm{g})$, ENR $(5 \mu \mathrm{g})$, FOX $(30 \mu \mathrm{g})$, MAR $(5 \mu \mathrm{g})$, OX $(1 \mu \mathrm{g}), \mathrm{P}$ $(10 \mathrm{U})$, and SXT $(25 \mu \mathrm{g})$. Clinical breakpoints from the Societé Française de Microbiology [24] were used for EFT $(30 \mu \mathrm{g})$. Results for CVN (30 $\mu \mathrm{g})$ were interpreted according to the manufacturer guidelines. As seen in the human
European Antimicrobial Resistance Surveillance Network (EARS-Net) report [18], when data on minimal inhibitory concentrations or inhibition zone diameter were not available, the laboratories' own interpretations (susceptible, intermediate and resistant) were accepted. This was the case for Spain, Serbia, and Germany that used contemporary CLSI guidelines, for the United Kingdom that used the breakpoints from the British Society of Antimicrobial Chemotherapy [25] and for the Netherlands and Switzerland (2008-2010) that used breakpoints recommended by the Dutch Committee on Guidelines for Susceptibility testing [26]. 


\section{Data analysis and statistical methods}

Statistical analysis was performed using the SAS statistical software package for Windows, version 9.3, (SAS Institute Inc, Cary, North Carolina, USA).

The Fisher exact test was used to compare pathogen frequencies by species or gender of the host, by simple/ multiple infection and by country. An alpha value of 0.05 was used.

Isolates were considered fully resistant when found to be resistant according to the clinical breakpoint applied. An isolate was considered susceptible when found to be susceptible or intermediate according to the clinical breakpoint applied. The antimicrobials included in this study are known to be highly concentrated in the urine so their report as susceptible may be appropriated for isolates categorized as intermediate [19].

Regarding third generation cephalosporins (3GC), laboratories tested different $3 \mathrm{GC}$ resistance surrogates. Therefore, to evaluate the antimicrobial resistance to $3 \mathrm{GC}$, an isolate was considered as $3 \mathrm{GC}$ resistant when it was resistant to at least one of the five $3 G C$ tested (CTX, CAZ, CVN, EFT or CPD). The same rational was applied to evaluate resistance to fluoroquinolones (FLU), namely using ENR, CIP or MAR as a marker of resistance. Methicillin-resistance in staphylococci was determined according to CLSI guidelines [23] using cefoxitin or oxacillin to evaluate resistance depending on the bacterial species considered. Yet, Germany and Spain did not send data on methicillin-resistance. France did not test staphylococci against oxacillin and thus did not report methicillin-resistance regarding Staphylococcus pseudintermedius (MRSP). The Netherlands did not have data on staphylococci susceptibility to OX or FOX but instead reported data on the detection of the mecA gene by PCR. The frequency of methicillin-resistance did not include staphylococci only identified to the genus level.

Enterobacteriaceae were considered multidrug-resistant (MDR) when fully resistant to three or more categories of antimicrobials, namely AMC, 3GC, SXT, CN and/or FLU. Unlike the MDR definition proposed by other authors [27], intermediate isolates from this study were considered as susceptible. This difference was applied because we are considering drugs that can be highly concentrated in urine. Furthermore, this approach will reduce any overestimation of MDR frequency due to the use of different breakpoint guidelines. Full-susceptibility (FullS) was defined as an isolate being susceptible for all the above-mentioned categories of antimicrobials. Since Belgium had no data available on 3GC and the Netherlands had little data on CN, MDR and FullS percentages do not include resistance to $3 \mathrm{GC}$ for Belgium and resistance to $\mathrm{CN}$ for the Netherlands.

As a rule, statistical analysis was only done when at least ten isolates for a specific organism-antimicrobial agent combination were reported for a given country. All frequencies are presented with a confidence interval of $95 \%(95 \% \mathrm{CI})$.

Maps of European resistance distribution were drawn considering the percentage of fully resistant isolates to the considered antimicrobial agent, over the years 2012-2013. A scale of colours was applied composed of six resistance intervals after the example of EARS-Net surveillance program reports [18].

Statistical analysis of temporal trends of antimicrobial resistance for a specific organism-antimicrobial agent combination were determined within each country. Temporal trends were only determined for countries reporting data on at least three consecutive years and ten isolates per year. A SAS LOGISTIC regression, with the year as a continuous variable and an alpha value of 0.05 was conducted. Temporal trends of resistance were mainly determined for E. coli since this was the most represented bacterial species. Yet, temporal trends of AMC, FLU and SXT in Proteus spp. were also determined for Belgium, France, the Netherlands and Sweden.

\section{Results}

Overall, data on 22,256 uropathogenic bacteria were obtained from 15,097 dog and 5963 cat positive urine cultures. Table 1 summarises the numbers of bacterial isolates obtained by year and country.

Considering the records containing information about the age, dogs $(n=4425)$ and cats $(n=1514)$ had similar

Table 1 Total number of isolated bacteria by year and country

\begin{tabular}{llllllll}
\hline Country $^{\text {a }}$ & Year & & & & & \\
\cline { 2 - 6 } & 2008 & 2009 & 2010 & 2011 & 2012 & 2013 & Total \\
\hline AT & - & - & - & - & 144 & 185 & 329 \\
BE & - & - & 547 & 578 & 623 & 739 & 2487 \\
DK & 29 & 30 & 53 & 116 & 153 & 205 & 587 \\
FR & - & - & 620 & 733 & 780 & 995 & 3128 \\
DE & - & 64 & 93 & 140 & 161 & 146 & 604 \\
EL & 24 & 29 & 13 & 11 & 32 & 43 & 152 \\
IT & - & 36 & 29 & 36 & 77 & 65 & 243 \\
NL & 480 & 867 & 958 & 1132 & 1195 & 1307 & 5939 \\
PT & 77 & 54 & 57 & 34 & 32 & 45 & 299 \\
RS & 17 & 19 & 10 & 2 & 3 & 3 & 54 \\
ES & 14 & 23 & 27 & 40 & 47 & 79 & 230 \\
SE & 730 & 924 & 1071 & 1202 & 1355 & 1647 & 6929 \\
CH & 109 & 120 & 112 & 125 & 114 & 174 & 754 \\
UK & 31 & 44 & 81 & 117 & 126 & 122 & 521 \\
Total & 1511 & 2210 & 3671 & 4267 & 4842 & 5755 & 22256 \\
\hline
\end{tabular}

${ }^{a} A T$, Austria; BE, Belgium; DK, Denmark; FR, France; DE, Germany; EL, Greece; IT, Italy; NL, the Netherlands; PT, Portugal; RS, Serbia; ES, Spain; SE, Sweden; $\mathrm{CH}$, Switzerland; UK, United Kingdom 
mean ages, namely 8.77 years $(\mathrm{SD} \pm 4.04,9.00$ median, $6.00 \mathrm{IQR}$, range $0.1-20)$ and 8.82 years $(\mathrm{SD} \pm 5.03,8.50$ median, 8.1 IQR, range 0.2-22) respectively. Gender was only specified in 3885 records where $61.41 \%$ (95 \% CI $59.69-63.12 \%, n=1900 / 3094)$ of dogs and $48.29 \%$ (95\% CI $44.81-51.78 \%, n=382 / 791$ ) of cats were females.

Among all urine cultures, 94.64 \% (95 \% CI 94.3394.94 \%, $n=19932 / 21060$ ) resulted in the growth of bacterial pure cultures, with no significant difference between cats and $\operatorname{dogs}(P=0.1856)$. Both in dogs and cats, E. coli was the most frequently identified bacteria and accounted for $59.45 \%$ (95 \% CI 58.80-60.09\%, $n=13231$ / 22256) of all isolates. The frequency of the remaining bacterial species differed significantly between dogs and cats (Table 2). Enterococcus spp. and Staphylococcus spp. frequencies were higher in cats, whereas Proteus spp. and Klebsiella spp. were more prevalent in dogs.

Considering the years 2012-2013, the major differences in E. coli (Table 3, Fig. 2) and Proteus spp. (Table 4, Fig. 3) antimicrobial resistance frequencies were seen between Northern (Denmark and Sweden) and Southern (Italy, Greece, Portugal and Spain) countries.

The lowest frequencies of AMC resistance in E. coli were detected in isolates from Denmark $(2.88 \%)$ and Belgium (4.29 \%). E. coli from Portugal (48.15 \%) had a significantly higher AMC resistance frequency $(P<0.05)$ when compared with all countries except for Spain, Italy and Greece (Table 3). Less than $15 \%$ of Proteus spp. were resistant to AMC in all countries with exception of Portugal (50 \%) and Spain (26.67 \%) (Table 4).

E. coli resistance to $3 \mathrm{GC}$ had a similar distribution to what was seen for AMC (Fig. 2). The highest 3GC resistance frequencies were found in Southern countries, namely Portugal (31.25\%), Italy (24.64 \%) and Spain (21.15 \%) (Table 3). Proteus spp. 3GC resistance was lower than 5 \% in Austria, Denmark, Sweden, Switzerland, United Kingdom and the Netherlands, whereas Portugal $(33.33 \%)$ and Spain $(15.38 \%)$ were the countries with the highest resistance levels (Table 4).

SXT resistance in Southern countries was higher than 25 and $45 \%$ for E. coli and Proteus spp., respectively (Tables 3 and 4; Figs. 2 and 3). Sweden and Denmark had the lowest SXT resistance values (lower than $9 \%$ ). The remaining included countries had frequencies ranging between $10.21-21.13 \%$ and $20-37.93 \%$ in E. coli and Proteus spp., respectively (Tables 3 and 4).

E. coli FLU resistance was higher in the Southern countries and ranged from $29.03 \%$ in Portugal to $31.88 \%$ in Italy (Table 3). Concerning Proteus spp., Spain and Germany had around 50 \% FLU resistance, followed by Italy and Portugal with around $40 \%$ (Table 4). Sweden, Denmark, Belgium and the Netherlands had less than 10 \% FLU resistant E. coli. Denmark, Sweden, United Kingdom and the Netherlands had less than 10 \% FLU resistant Proteus spp. (Figs. 2 and 3).

Table 2 Uropathogenic bacteria aetiology, single versus mixed infections and cat versus dog as host species

\begin{tabular}{|c|c|c|c|c|c|c|c|c|c|c|c|c|}
\hline \multirow[t]{2}{*}{ Organism } & \multicolumn{2}{|c|}{ Overall } & \multicolumn{2}{|c|}{ Single organism } & \multicolumn{2}{|c|}{ Mixed infections } & \multirow[b]{2}{*}{$P$} & \multicolumn{2}{|l|}{ Dogs } & \multicolumn{2}{|l|}{ Cats } & \multirow[b]{2}{*}{$P$} \\
\hline & $n$ & $\begin{array}{l}\% \\
(95 \% C l)^{a}\end{array}$ & $n$ & $\begin{array}{l}\% \\
(95 \% C l)^{a}\end{array}$ & $n$ & $\begin{array}{l}\% \\
(95 \% C l)^{a}\end{array}$ & & $n$ & $\begin{array}{l}\% \\
(95 \% C l)^{a}\end{array}$ & $n$ & $\begin{array}{l}\% \\
(95 \% C l)^{a}\end{array}$ & \\
\hline Enterobacter spp. & 308 & $\begin{array}{l}1.38 \\
(1.23-1.54)\end{array}$ & 244 & $\begin{array}{l}1.22 \\
(1.07-1.38)\end{array}$ & 64 & $\begin{array}{l}2.75 \\
(2.09-3.42)\end{array}$ & $<0.0001$ & 194 & $\begin{array}{l}1.21 \\
(1.04-1.38)\end{array}$ & 114 & $\begin{array}{l}1.81 \\
(1.48-2.14)\end{array}$ & 0.0008 \\
\hline Enterococcus spp. & 1506 & $\begin{array}{l}6.77 \\
(6.44-7.10)\end{array}$ & 1129 & $\begin{array}{l}5.66 \\
(5.34-5.99)\end{array}$ & 377 & $\begin{array}{l}16.22 \\
(14.72-17.72)\end{array}$ & $<0.0001$ & 745 & $\begin{array}{l}4.66 \\
(4.34-4.99)\end{array}$ & 761 & $\begin{array}{l}12.11 \\
(11.31-12.92)\end{array}$ & $<0.0001$ \\
\hline Escherichia coli & 13231 & $\begin{array}{l}59.45 \\
(58.80-60.09)\end{array}$ & 12417 & $\begin{array}{l}62.30 \\
(61.62-62.97)\end{array}$ & 814 & $\begin{array}{l}35.03 \\
(33.09-36-97)\end{array}$ & $<0.0001$ & 9506 & $\begin{array}{l}59.51 \\
(58.75-60.27)\end{array}$ & 3725 & $\begin{array}{l}59.30 \\
(58.08-60.51)\end{array}$ & 0.7832 \\
\hline Klebsiella spp. & 478 & $\begin{array}{l}2.15 \\
(1.96-2.34)\end{array}$ & 400 & $\begin{array}{l}2.01 \\
(1.81-2.20)\end{array}$ & 78 & $\begin{array}{l}3.36 \\
(2.62-4.09)\end{array}$ & $<0.0001$ & 385 & $\begin{array}{l}2.41 \\
(2.17-2.65)\end{array}$ & 93 & $\begin{array}{l}1.48 \\
(1.18-1.78)\end{array}$ & $<0.0001$ \\
\hline Proteus spp. & 1992 & $\begin{array}{l}8.95 \\
(8.58-9.33)\end{array}$ & 1770 & $\begin{array}{l}8.88 \\
(8.49-9.28)\end{array}$ & 222 & $\begin{array}{l}9.55 \\
(8.36-10.75)\end{array}$ & 0.2824 & 1869 & $\begin{array}{l}11.70 \\
(1.20-1.22)\end{array}$ & 123 & $\begin{array}{l}1.96 \\
(1.62-2.30)\end{array}$ & $<0.0001$ \\
\hline Pseudomonas spp. & 389 & $\begin{array}{l}1.75 \\
(1.58-1.92)\end{array}$ & 315 & $\begin{array}{l}1.58 \\
(1.41-1.75)\end{array}$ & 74 & $\begin{array}{l}3.18 \\
(2.47-3.90)\end{array}$ & $<0.0001$ & 293 & $\begin{array}{l}1.83 \\
(1.63-2.04)\end{array}$ & 96 & $\begin{array}{l}1.53 \\
(1.22-1.83)\end{array}$ & 0.1249 \\
\hline Staphylococcus spp. & 2893 & $\begin{array}{l}13.00 \\
(12.56-13.44)\end{array}$ & 2519 & $\begin{array}{l}12.64 \\
(12.18-13.10)\end{array}$ & 374 & $\begin{array}{l}16.09 \\
(14.60-17.59)\end{array}$ & $<0.0001$ & 1836 & $\begin{array}{l}11.49 \\
(11.00-11.99)\end{array}$ & 1057 & $\begin{array}{l}16.83 \\
(15.90-17.75)\end{array}$ & $<0.0001$ \\
\hline Streptococcus spp. & 802 & $\begin{array}{l}3.60 \\
(3.36-3.85)\end{array}$ & 586 & $\begin{array}{l}2.94 \\
(2.71-3.17)\end{array}$ & 216 & $\begin{array}{l}9.29 \\
(8.11-10.47)\end{array}$ & $<0.0001$ & 675 & $\begin{array}{l}4.23 \\
(3.91-4.54)\end{array}$ & 127 & $\begin{array}{l}2.02 \\
(1.67-2.37)\end{array}$ & $<0.0001$ \\
\hline Other & 657 & $\begin{array}{l}2.95 \\
(2.73-3.17)\end{array}$ & 552 & $\begin{array}{l}2.77 \\
(2.54-3.00)\end{array}$ & 105 & $\begin{array}{l}4.52 \\
(3.67-5.36)\end{array}$ & - & 471 & $\begin{array}{l}2.95 \\
(2.69-3.21)\end{array}$ & 186 & $\begin{array}{l}2.96 \\
(2.54-3.38)\end{array}$ & - \\
\hline
\end{tabular}

${ }^{\mathrm{a}} 95 \% \mathrm{Cl}, 95 \%$ Confidence interval

$n$ - Total number of isolates

$P$ - $P$ value obtained by Fisher exact test when comparing single versus mixed infections and cat versus dog as host. Statistically significant values are highlighted in bold 
Table 3 Percentage of resistance in Escherichia coli by antimicrobial and country in 2012-2013

\begin{tabular}{|c|c|c|c|c|c|c|c|c|c|c|c|c|c|}
\hline \multirow[t]{2}{*}{$\overline{\text { Country }^{\mathrm{a}}}$} & \multicolumn{2}{|l|}{ AMC } & \multicolumn{2}{|l|}{$3 G C$} & \multicolumn{2}{|l|}{ FLU } & \multicolumn{2}{|l|}{$C N$} & \multicolumn{2}{|l|}{ SXT } & \multicolumn{3}{|c|}{ Combined resistance } \\
\hline & $n$ & $\begin{array}{l}\% \mathrm{R} \\
(95 \% \mathrm{Cl})^{\mathrm{b}} \\
\text { [Stat. Dif.] }^{\mathrm{c}}\end{array}$ & $n$ & $\begin{array}{l}\% \mathrm{R} \\
(95 \% \mathrm{Cl})^{\mathrm{b}} \\
\text { [Stat. Dif.] }^{\mathrm{c}}\end{array}$ & $n$ & $\begin{array}{l}\% \mathrm{R} \\
(95 \% \mathrm{Cl})^{\mathrm{b}} \\
\text { [Stat. Dif.] }^{\mathrm{c}}\end{array}$ & $n$ & $\begin{array}{l}\% \mathrm{R} \\
(95 \% \mathrm{Cl})^{\mathrm{b}} \\
\text { [Stat. Dif.] }^{\mathrm{c}}\end{array}$ & $n$ & $\begin{array}{l}\% \text { R } \\
(95 \% \text { Cl) } \\
\text { [Stat. Dif.] }]^{c}\end{array}$ & $n$ & $\begin{array}{l}\text { \% MDR } \\
(95 \% \text { Cl) } \\
\text { [Stat. Dif.] }]^{\mathrm{c}}\end{array}$ & $\begin{array}{l}\text { \% FullS } \\
(95 \% \text { Cl) } \\
\text { [Stat. Dif.] }\end{array}$ \\
\hline AT & 142 & $\begin{array}{l}14.08 \\
(8.36-19.81) \\
{[a, b]}\end{array}$ & 142 & $\begin{array}{l}5.63 \\
(1.84-9.43) \\
{[a, b]}\end{array}$ & 142 & $\begin{array}{l}11.97 \\
(6.63-17.31) \\
{[a]}\end{array}$ & 142 & $\begin{array}{l}5.63 \\
(1.84-9.43) \\
{[a, b]}\end{array}$ & 142 & $\begin{array}{l}14.08 \\
(8.36-19.81) \\
{[a, b]}\end{array}$ & 142 & $\begin{array}{l}8.45 \\
(3.88-13.03) \\
{[a]}\end{array}$ & $\begin{array}{l}78.87 \\
(72.16-85.59) \\
\text { [a] }\end{array}$ \\
\hline $\mathrm{BE}$ & 840 & $\begin{array}{l}4.29 \\
(2.92-5.66) \\
{[c]}\end{array}$ & 0 & - & 769 & $\begin{array}{l}6.63 \\
(4.87-8.39) \\
{[b]}\end{array}$ & 840 & $\begin{array}{l}1.67 \\
(0.80-2.53) \\
{[c]}\end{array}$ & 839 & $\begin{array}{l}10.37 \\
(8.31-12.43) \\
{[a]}\end{array}$ & $769^{d}$ & $\begin{array}{l}1.43^{\mathrm{d}} \\
(0.59-2.27) \\
-\end{array}$ & $\begin{array}{l}85.05^{\mathrm{d}} \\
(82.52-87.57) \\
-\end{array}$ \\
\hline DK & 206 & $\begin{array}{l}2.88 \\
(0.61-5.16) \\
{[c]}\end{array}$ & 208 & $\begin{array}{l}4.33 \\
(1.41-7.09) \\
{[a, c]}\end{array}$ & 208 & $\begin{array}{l}2.88 \\
(0.61-5.16) \\
{[c]}\end{array}$ & 208 & $\begin{array}{l}1.92 \\
(0.06-3.79) \\
{[a, c]}\end{array}$ & 208 & $\begin{array}{l}8.17 \\
(4.45-11.90) \\
{[a, c]}\end{array}$ & 208 & $\begin{array}{l}2.88 \\
(0.61-5.16) \\
{[b]}\end{array}$ & $\begin{array}{l}88.94 \\
(84.68-93.20) \\
{[b, c]}\end{array}$ \\
\hline$F R$ & 954 & $\begin{array}{l}12.79 \\
(10.67-15.91) \\
{[a, d]}\end{array}$ & 933 & $\begin{array}{l}10.83 \\
(8.83-12.82) \\
{[b]}\end{array}$ & 948 & $\begin{array}{l}12.76 \\
(10.64-14.89) \\
{[a]}\end{array}$ & 951 & $\begin{array}{l}3.36 \\
(2.22-4.51) \\
{[a, d]}\end{array}$ & 959 & $\begin{array}{l}16.27 \\
(13.93-18.60) \\
{[b, d]}\end{array}$ & 909 & $\begin{array}{l}11.00 \\
(8.97-13.04) \\
{[a, c]}\end{array}$ & $\begin{array}{l}77.23 \\
(74.50-79.95) \\
{[a]}\end{array}$ \\
\hline $\mathrm{DE}$ & 153 & $\begin{array}{l}11.76 \\
(6.66-16.87) \\
{[a, d]}\end{array}$ & 152 & $\begin{array}{l}11.84 \\
(6.71-16.98) \\
{[b, d]}\end{array}$ & 153 & $\begin{array}{l}16.34 \\
(10.48-22.20) \\
{[a, d]}\end{array}$ & 153 & $\begin{array}{l}1.96 \\
(0.00-4.16) \\
{[a, c, d]}\end{array}$ & 153 & $\begin{array}{l}17.65 \\
(11.61-23.69) \\
{[b, d, e]}\end{array}$ & 152 & $\begin{array}{l}8.55 \\
(4.11-13.00) \\
{[a]}\end{array}$ & $\begin{array}{l}67.76 \\
(60.33-75.19) \\
\text { [d] }\end{array}$ \\
\hline EL & 31 & $\begin{array}{l}25.81 \\
(10.40-41.21) \\
{[b, d, e, f]}\end{array}$ & 9 & $\begin{array}{l}7 R / 2 S \\
-\end{array}$ & 30 & $\begin{array}{l}30.00 \\
(13.60-46.40) \\
{[d, e]}\end{array}$ & 0 & - & 26 & $\begin{array}{l}34.62 \\
(16.33-52.90) \\
{[e, f]}\end{array}$ & 0 & - & - \\
\hline IT & 69 & $\begin{array}{l}26.09 \\
(15.73-36.45) \\
{[e, f]}\end{array}$ & 69 & $\begin{array}{l}24.64 \\
(14.47-34.80) \\
{[e]}\end{array}$ & 69 & $\begin{array}{l}31.88 \\
(20.89-42.88) \\
{[\mathrm{e}]}\end{array}$ & 69 & $\begin{array}{l}14.49 \\
(6.19-22.80) \\
{[\mathrm{e}]}\end{array}$ & 69 & $\begin{array}{l}28.99 \\
(18.28-39.69) \\
{[e, f]}\end{array}$ & 69 & $\begin{array}{l}28.99 \\
(18.28-39.69) \\
{[d]}\end{array}$ & $\begin{array}{l}63.77 \\
(52.43-75.11) \\
{[d, e, f]}\end{array}$ \\
\hline $\mathrm{NL}$ & 1461 & $\begin{array}{l}10.81 \\
(9.22-12.41) \\
{[a]}\end{array}$ & 1380 & $\begin{array}{l}3.77 \\
(2.76-4.77) \\
{[a, c]}\end{array}$ & 1457 & $\begin{array}{l}4.94 \\
(3.83-6.05) \\
{[b, c]}\end{array}$ & 81 & $\begin{array}{l}3.70 \\
(0.00-7.82) \\
{[a, c, f]}\end{array}$ & 1459 & $\begin{array}{l}10.21 \\
(8.66-11.77) \\
{[a]}\end{array}$ & $1380^{d}$ & $\begin{array}{l}2.25^{\mathrm{d}} \\
(1.46-3.03) \\
-\end{array}$ & $\begin{array}{l}81.30^{d} \\
(79.25-83.36) \\
-\end{array}$ \\
\hline PT & 27 & $\begin{array}{l}48.15 \\
(29.30-66.99) \\
{[e]}\end{array}$ & 32 & $\begin{array}{l}31.25 \\
(15.19-47.31) \\
{[e]}\end{array}$ & 31 & $\begin{array}{l}29.03 \\
(13.05-45.01) \\
{[d, e]}\end{array}$ & 30 & $\begin{array}{l}10.00 \\
(0.00-20.74) \\
{[b, d, e, f]}\end{array}$ & 31 & $\begin{array}{l}32.26 \\
(15.80-48.71) \\
{[e, f]}\end{array}$ & 25 & $\begin{array}{l}24.00 \\
(7.26-40.74) \\
{[c, d, e]}\end{array}$ & $\begin{array}{l}32.00 \\
(13.71-50.29) \\
{[g, h]}\end{array}$ \\
\hline RS & 3 & $2 R / 1 S-$ & 2 & $\begin{array}{l}1 \mathrm{R} / 1 \mathrm{~S} \\
-\end{array}$ & 3 & $\begin{array}{l}0 R / 3 S \\
-\end{array}$ & 3 & $0 R / 3 S$ & 3 & $\begin{array}{l}1 R / 2 S \\
-\end{array}$ & 2 & $\begin{array}{l}1 \mathrm{MDR} \\
-\end{array}$ & 1Full-S \\
\hline ES & 60 & $\begin{array}{l}31.67 \\
(19.90-43.44) \\
{[e, f]}\end{array}$ & 52 & $\begin{array}{l}21.15 \\
(10.05-32.25) \\
{[d, e]}\end{array}$ & 61 & $\begin{array}{l}29.51 \\
(18.06-40.95) \\
{[\mathrm{e}]}\end{array}$ & 46 & $\begin{array}{l}15.22 \\
(4.84-25.60) \\
{[b, e]}\end{array}$ & 60 & $\begin{array}{l}26.67 \\
(15.48-37.86) \\
{[e, f]}\end{array}$ & 37 & $\begin{array}{l}29.73 \\
(15.00-44.46) \\
{[d, e]}\end{array}$ & $\begin{array}{l}43.24 \\
(27.28-59.21) \\
{[\mathrm{e}, \mathrm{g}]}\end{array}$ \\
\hline SE & 2091 & $\begin{array}{l}6.98 \\
(5.89-8.07) \\
{[g]}\end{array}$ & 2082 & $\begin{array}{l}0 \\
{[\mathrm{f}]}\end{array}$ & 2091 & $\begin{array}{l}1.05 \\
(0.61-1.49) \\
{[f]}\end{array}$ & 2091 & $\begin{array}{l}0.19 \\
(0.00-0.38) \\
{[g]}\end{array}$ & 2091 & $\begin{array}{l}4.97 \\
(4.04-5.91) \\
{[c]}\end{array}$ & 2082 & $\begin{array}{l}0.24 \\
(0.03-0.45) \\
{[f]}\end{array}$ & $\begin{array}{l}90.2 \\
(88.92-91.48) \\
{[b, h]}\end{array}$ \\
\hline $\mathrm{CH}$ & 133 & $\begin{array}{l}10.53 \\
(5.31-15.74) \\
{[a, g]}\end{array}$ & 133 & $\begin{array}{l}8.27 \\
(3.59-12.95) \\
{[b, c]}\end{array}$ & 132 & $\begin{array}{l}13.64 \\
(7.78-19.49) \\
{[a, d]}\end{array}$ & 132 & $\begin{array}{l}6.82 \\
(2.52-11.12) \\
{[b, d, e, f]}\end{array}$ & 131 & $\begin{array}{l}13.74 \\
(7.85-19.64) \\
{[a, d, g]}\end{array}$ & 130 & $\begin{array}{l}10.00 \\
(4.84-15.16) \\
{[a, e]}\end{array}$ & $\begin{array}{l}83.08 \\
(76.63-89.52) \\
{[a, c]}\end{array}$ \\
\hline UK & 143 & $\begin{array}{l}21.68 \\
(14.92-28.43) \\
{[b, f]}\end{array}$ & 143 & $\begin{array}{l}20.98 \\
(14.31-27.65) \\
{[d, e]}\end{array}$ & 143 & $\begin{array}{l}11.89 \\
(6.58-17.19) \\
{[a]}\end{array}$ & 92 & $\begin{array}{l}6.52 \\
(1.48-11.57) \\
{[a, e, f]}\end{array}$ & 142 & $\begin{array}{l}21.13 \\
(14.41-27.84) \\
{[b, f, g]}\end{array}$ & 89 & $\begin{array}{l}15.56 \\
(8.07-23.04) \\
{[a, e]}\end{array}$ & $\begin{array}{l}67.78 \\
(58.12-77.43) \\
{[a, d, f]}\end{array}$ \\
\hline
\end{tabular}

$A M C$ amoxicillin clavulanate, $3 G C$ third generation cephalosporins, FLU fluoroquinolones, CN gentamicin, SXT trimethoprim/sulfamethoxazole, MDR multidrug-resistant, Full-S fully-susceptible

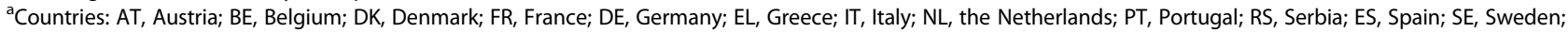
$\mathrm{CH}$, Switzerland; UK, United Kingdom.

${ }^{b} 95 \% \mathrm{Cl}_{1} 95 \%$ Confidence interval

'Stat. Dif., Statistical significant differences. Countries with no statistical difference are marked with the same letter. Countries were compared by Fisher exact test with an alpha value of 0.05 . Countries with less than ten tested isolates were not compared. Regarding MDR and FullS, only countries tested for all the considered antimicrobials were compared

$n$, Total number of Escherichia coli tested for the considered antimicrobial category

${ }^{d}$ MDR and FullS percentages do not include resistance to $3 G C$ for Belgium and resistance to $C N$ for the Netherlands

Overall, E. coli $\mathrm{CN}$ resistance was lower than $16 \%$ (Table 3). Regarding resistance to $\mathrm{CN}$ in Proteus spp. the same resistance frequency occurred with the exception of Portugal where a higher resistance frequency was recorded (33.33\%) (Table 4).
E. coli from Portugal, Spain and Italy, and Proteus spp. from Portugal showed the highest frequencies of MDR (Tables 3 and 4). As expected, Portugal was one of the countries with lowest FullS, both in E. coli (32.00 \%) and Proteus spp. (35.71 \%). Italy had even lower FullS in 


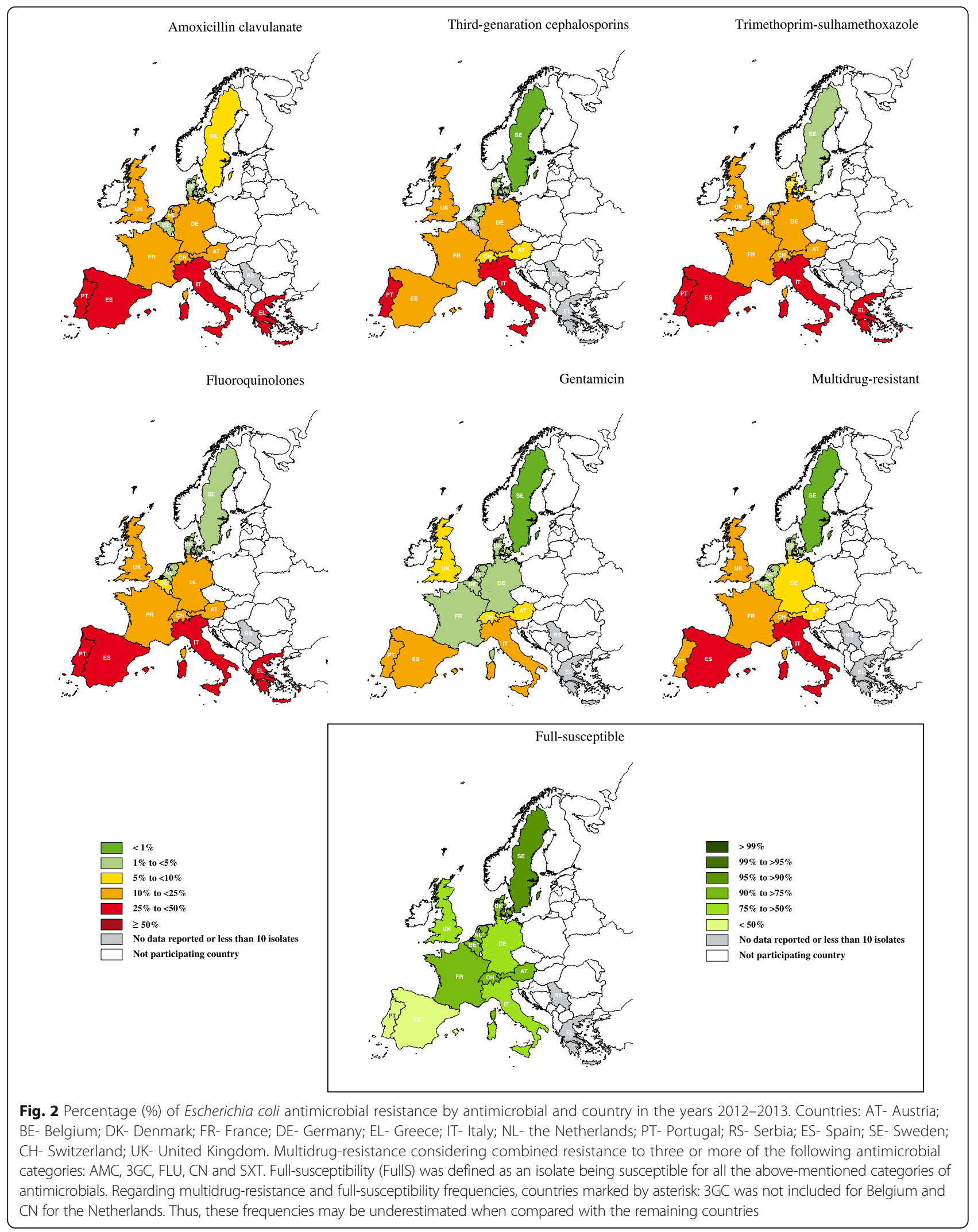


Table 4 Percentage of resistance in Proteus spp. by antimicrobial and country in 2012-2013

\begin{tabular}{|c|c|c|c|c|c|c|c|c|c|c|c|c|c|}
\hline \multirow[t]{2}{*}{ Country $^{a}$} & \multicolumn{2}{|c|}{ AMC } & \multicolumn{2}{|l|}{$3 G C$} & \multicolumn{2}{|l|}{ FLU } & \multicolumn{2}{|l|}{$\mathrm{CN}$} & \multicolumn{2}{|l|}{ SXT } & \multicolumn{3}{|c|}{ Combined resistance } \\
\hline & n & $\begin{array}{l}\% \mathrm{R} \\
(95 \% \mathrm{Cl})^{\mathrm{b}} \\
\text { [Stat. Dif.] }^{\mathrm{c}}\end{array}$ & n & $\begin{array}{l}\% \mathrm{R} \\
(95 \% \mathrm{Cl})^{\mathrm{b}} \\
\text { [Stat. Dif.] }^{\mathrm{c}}\end{array}$ & $\bar{n}$ & $\begin{array}{l}\% \text { R } \\
(95 \% \text { Cl) } \\
\text { [Stat. Dif.] }^{c}\end{array}$ & $\bar{n}$ & $\begin{array}{l}\% \text { R } \\
(95 \% \text { Cl) })^{\mathrm{b}} \\
\text { [Stat. Dif.] }^{\mathrm{c}}\end{array}$ & n & $\begin{array}{l}\% \text { R } \\
(95 \% \text { Cl) } \\
\text { [Stat. Dif.] }^{c}\end{array}$ & $\bar{n}$ & $\begin{array}{l}\% \text { MDR }(95 \% \mathrm{Cl})^{\mathrm{b}} \\
\text { [Stat. Dif.] }^{\mathrm{c}}\end{array}$ & $\begin{array}{l}\text { \% FullS } \\
(95 \% \text { Cl) } \\
\text { [Stat. Dif.] }^{c}\end{array}$ \\
\hline$\overline{\mathrm{AT}}$ & 29 & $\begin{array}{l}10.34 \\
(0.00-21.43) \\
{[a, b, c]}\end{array}$ & 29 & $\begin{array}{l}0 \\
{[a, b]}\end{array}$ & 29 & $\begin{array}{l}17.24 \\
(3.49-30.99) \\
{[a, b, c]}\end{array}$ & 29 & $\begin{array}{l}6.90 \\
(0.00-16.12) \\
{[a, b]}\end{array}$ & 29 & $\begin{array}{l}37.93 \\
(20.27-55.59) \\
{[a, b]}\end{array}$ & 29 & $\begin{array}{l}6.90 \\
(0.00-16.12) \\
{[a]}\end{array}$ & $\begin{array}{l}55.17 \\
(37.07-73.27) \\
{[a, b]}\end{array}$ \\
\hline $\mathrm{BE}$ & 143 & $\begin{array}{l}2.10 \\
(0.00-4.45) \\
{[a, g]}\end{array}$ & 0 & $\begin{array}{l}- \\
-\end{array}$ & 135 & $\begin{array}{l}28.15 \\
(20.56-35.73) \\
{[a, d]}\end{array}$ & 155 & $\begin{array}{l}9.68 \\
(5.02-14.33) \\
{[a]}\end{array}$ & 154 & $\begin{array}{l}35.06 \\
(27.53-42.60) \\
{[a, b]}\end{array}$ & $125^{d}$ & $\begin{array}{l}4.80^{d} \\
(1.05-8.55) \\
-\end{array}$ & $\begin{array}{l}57.60^{d} \\
(48.94-66.26) \\
-\end{array}$ \\
\hline DK & 31 & $\begin{array}{l}0 \\
{[a, d]}\end{array}$ & 31 & $\begin{array}{l}0 \\
{[a, b]}\end{array}$ & 31 & $\begin{array}{l}0 \\
{[e, f]}\end{array}$ & 31 & $\begin{array}{l}0 \\
{[a, b]}\end{array}$ & 31 & $\begin{array}{l}6.45 \\
(0.00-15.10) \\
{[c]}\end{array}$ & 31 & $\begin{array}{l}0 \\
{[a, b]}\end{array}$ & $\begin{array}{l}93.55 \\
(44.41-84.90) \\
{[c]}\end{array}$ \\
\hline$F R$ & 215 & $\begin{array}{l}7.44 \\
(3.93-10.95) \\
{[b, d]}\end{array}$ & 211 & $\begin{array}{l}6.64 \\
(3.28-9.99) \\
{[a]}\end{array}$ & 212 & $\begin{array}{l}17,92 \\
(12.76-23.09) \\
{[b, g]}\end{array}$ & 214 & $\begin{array}{l}9.81 \\
(5.83-13.80) \\
{[a]}\end{array}$ & 216 & $\begin{array}{l}27.78 \\
(21.80-33.75) \\
{[a]}\end{array}$ & 204 & $\begin{array}{l}10.29 \\
(6.12-14.46) \\
{[a]}\end{array}$ & $\begin{array}{l}66.67 \\
(60.20-73.14) \\
{[a]}\end{array}$ \\
\hline $\mathrm{DE}$ & 10 & $\begin{array}{l}0 \\
{[a, b, f]}\end{array}$ & 10 & $\begin{array}{l}10.00 \\
(0.00-28.59) \\
{[a, b, c]}\end{array}$ & 10 & $\begin{array}{l}50.00 \\
(19.01-80.99) \\
{[a, d]}\end{array}$ & 10 & $\begin{array}{l}0 \\
{[a, b, c]}\end{array}$ & 10 & $\begin{array}{l}20.00 \\
(0.00-44.79) \\
{[a, c, d]}\end{array}$ & 10 & $\begin{array}{l}0 \\
{[a, b]}\end{array}$ & $\begin{array}{l}50.00 \\
(19.01-80.99) \\
{[a, d]}\end{array}$ \\
\hline EL & 8 & $\begin{array}{l}1 \mathrm{R} / 7 \mathrm{~S} \\
-\end{array}$ & 0 & - & 8 & $\begin{array}{l}4 R / 4 S \\
-\end{array}$ & 0 & - & 7 & $\begin{array}{l}4 R / 3 R \\
-\end{array}$ & 0 & - & - \\
\hline IT & 12 & $\begin{array}{l}0 \\
{[a, b, c]}\end{array}$ & 12 & $\begin{array}{l}8.33 \\
(0.00-23.97) \\
{[a, b, c]}\end{array}$ & 12 & $\begin{array}{l}41.67 \\
(13.77-69.56) \\
{[a, d, g]}\end{array}$ & 12 & $\begin{array}{l}8.33 \\
(0.00-23.97) \\
{[a, b, c]}\end{array}$ & 12 & $\begin{array}{l}66.67 \\
(39.99-93.34) \\
{[b, e]}\end{array}$ & 12 & $\begin{array}{l}8.33 \\
(0.00-23.97) \\
{[a, b, c]}\end{array}$ & $\begin{array}{l}16.67 \\
(0.00-37.75) \\
{[d]}\end{array}$ \\
\hline $\mathrm{NL}$ & 261 & $\begin{array}{l}6.13 \\
(3.22-9.04) \\
{[a, b, e]}\end{array}$ & 244 & $\begin{array}{l}2.87 \\
(0.77-4.96) \\
{[a]}\end{array}$ & 260 & $\begin{array}{l}8.85 \\
(5.39-12.30) \\
{[c, e, h]}\end{array}$ & 17 & $\begin{array}{l}11.76 \\
(0.00-27.08) \\
{[a, c]}\end{array}$ & 260 & $\begin{array}{l}27.31 \\
(21.89-32.72) \\
{[a]}\end{array}$ & $243^{d}$ & $\begin{array}{l}3.29^{d} \\
(1.05-5.54) \\
-\end{array}$ & $\begin{array}{l}69.96^{d} \\
(64.19-75.72) \\
-\end{array}$ \\
\hline PT & 14 & $\begin{array}{l}50.00 \\
(23.81-76.19) \\
{[\mathrm{e}]}\end{array}$ & 15 & $\begin{array}{l}33.33 \\
(9.48-57.19) \\
{[c, d]}\end{array}$ & 15 & $\begin{array}{l}40.00 \\
(15.21-64.79) \\
{[a, g, i]}\end{array}$ & 15 & $\begin{array}{l}33.33 \\
(9.48-57.19) \\
{[c]}\end{array}$ & 15 & $\begin{array}{l}46.67 \\
(21.42-71.91) \\
{[a, e]}\end{array}$ & 14 & $\begin{array}{l}42.86 \\
(16.93-68.78) \\
{[c]}\end{array}$ & $\begin{array}{l}35.71 \\
(10.61-60.81) \\
{[b, d, e]}\end{array}$ \\
\hline RS & 1 & $\begin{array}{l}\mathrm{OR} / 1 \mathrm{~S} \\
-\end{array}$ & 1 & $\begin{array}{l}1 \mathrm{R} / \mathrm{OS} \\
-\end{array}$ & 1 & $\begin{array}{l}0 R / 1 S \\
-\end{array}$ & 1 & $\begin{array}{l}\mathrm{OR} / 1 \mathrm{~S} \\
-\end{array}$ & 0 & - & 0 & - & - \\
\hline ES & 15 & $\begin{array}{l}26.67 \\
(4.29-49.05) \\
{[c, e, f]}\end{array}$ & 13 & $\begin{array}{l}15.38 \\
(0.00-35.00) \\
{[a, d]}\end{array}$ & 15 & $\begin{array}{l}53.33 \\
(28.09-78.58) \\
{[d, i]}\end{array}$ & 9 & $2 \mathrm{R} / 7 \mathrm{~S}$ & 15 & $\begin{array}{l}53.33 \\
(28.09-78.58) \\
{[b, d, e]}\end{array}$ & 7 & 2MDR & $\begin{array}{l}\text { 4Full-S } \\
-\end{array}$ \\
\hline SE & 170 & $\begin{array}{l}2.35 \\
(0.07-4.63) \\
{[a, f, g]}\end{array}$ & 169 & $\begin{array}{l}0 \\
{[b, e]}\end{array}$ & 170 & $\begin{array}{l}0.59 \\
(0.00-1.74) \\
{[f]}\end{array}$ & 170 & $\begin{array}{l}0.59 \\
(0.00-1.74) \\
{[b]}\end{array}$ & 170 & $\begin{array}{l}7.06 \\
(3.21-10.91) \\
{[c]}\end{array}$ & 169 & $\begin{array}{l}0 \\
{[b]}\end{array}$ & $\begin{array}{l}91.12 \\
(86.84-95.41) \\
{[c]}\end{array}$ \\
\hline $\mathrm{CH}$ & 17 & $\begin{array}{l}0 \\
{[a, b, f]}\end{array}$ & 17 & $\begin{array}{l}0 \\
{[a, e]}\end{array}$ & 17 & $\begin{array}{l}23.53 \\
(3.37-43.69) \\
{[a, g, h, l, j]}\end{array}$ & 17 & $\begin{array}{l}5.88 \\
(0.00-17.07) \\
{[a, b, c]}\end{array}$ & 17 & $\begin{array}{l}35.29 \\
(12.58-58.01) \\
{[a, e]}\end{array}$ & 17 & $\begin{array}{l}0 \\
{[a, b]}\end{array}$ & $\begin{array}{l}64.71 \\
(41.99-87.42) \\
{[a, e]}\end{array}$ \\
\hline UK & 16 & $\begin{array}{l}12.50 \\
(0.00-28.70) \\
{[a, b, f]}\end{array}$ & 16 & $\begin{array}{l}0 \\
{[a, e]}\end{array}$ & 16 & $\begin{array}{l}0 \\
{[b, e, f, j]}\end{array}$ & 11 & $\begin{array}{l}0 \\
{[a, b, c]}\end{array}$ & 15 & $\begin{array}{l}33.33 \\
(9.48-57.19) \\
{[a, e]}\end{array}$ & 10 & $\begin{array}{l}0 \\
{[a, b]}\end{array}$ & $\begin{array}{l}70.00 \\
(41.60-98.40) \\
{[a, c, e]}\end{array}$ \\
\hline
\end{tabular}

AMC amoxicillin clavulanate, $3 G C$ third generation cephalosporins, FLU fluoroquinolones, CN gentamicin, SXT trimethoprim/sulfamethoxazole, MDR multidrug-resistant, FullS fully-susceptible

${ }^{a}$ Countries: AT, Austria; BE, Belgium; DK, Denmark; FR, France; DE, Germany; EL, Greece; IT, Italy; NL, the Netherlands; PT, Portugal; RS, Serbia; ES, Spain; SE, Sweden; $\mathrm{CH}$, Switzerland; UK, United Kingdom.

${ }^{\mathrm{b}} 95 \% \mathrm{Cl}, 95 \%$ Confidence interval.

'Stat. Dif., Statistical significant differences. Countries with no statistical difference are marked with the same letter. Countries were compared by Fisher exact test with an alpha value of 0.05 . Countries with less than ten tested isolates were not compared. Regarding MDR and FullS, only countries tested for all the considered antimicrobials were compared

$n$, Total number of Proteus spp. tested for the considered antimicrobial category

${ }^{d}$ MDR and FullS percentages do not include resistance to $3 G C$ for Belgium and resistance to $C N$ for the Netherlands

Proteus spp. (16.67 \%). Most of the remaining countries had MDR levels lower than $10 \%$, with the exception of MDR E. coli from United Kingdom (15.56 \%) and France (11 \%). The highest E. coli and Proteus spp. FullS frequencies were found in Denmark and Sweden (Tables 3 and 4).
Due to the limited number of Staphylococcus spp. isolates available, the percentage of resistance to antimicrobials was determined on fewer countries for this group of bacteria (Tables 5 and 6). In most countries, Staphylococcus pseudintermedius was the most frequently 


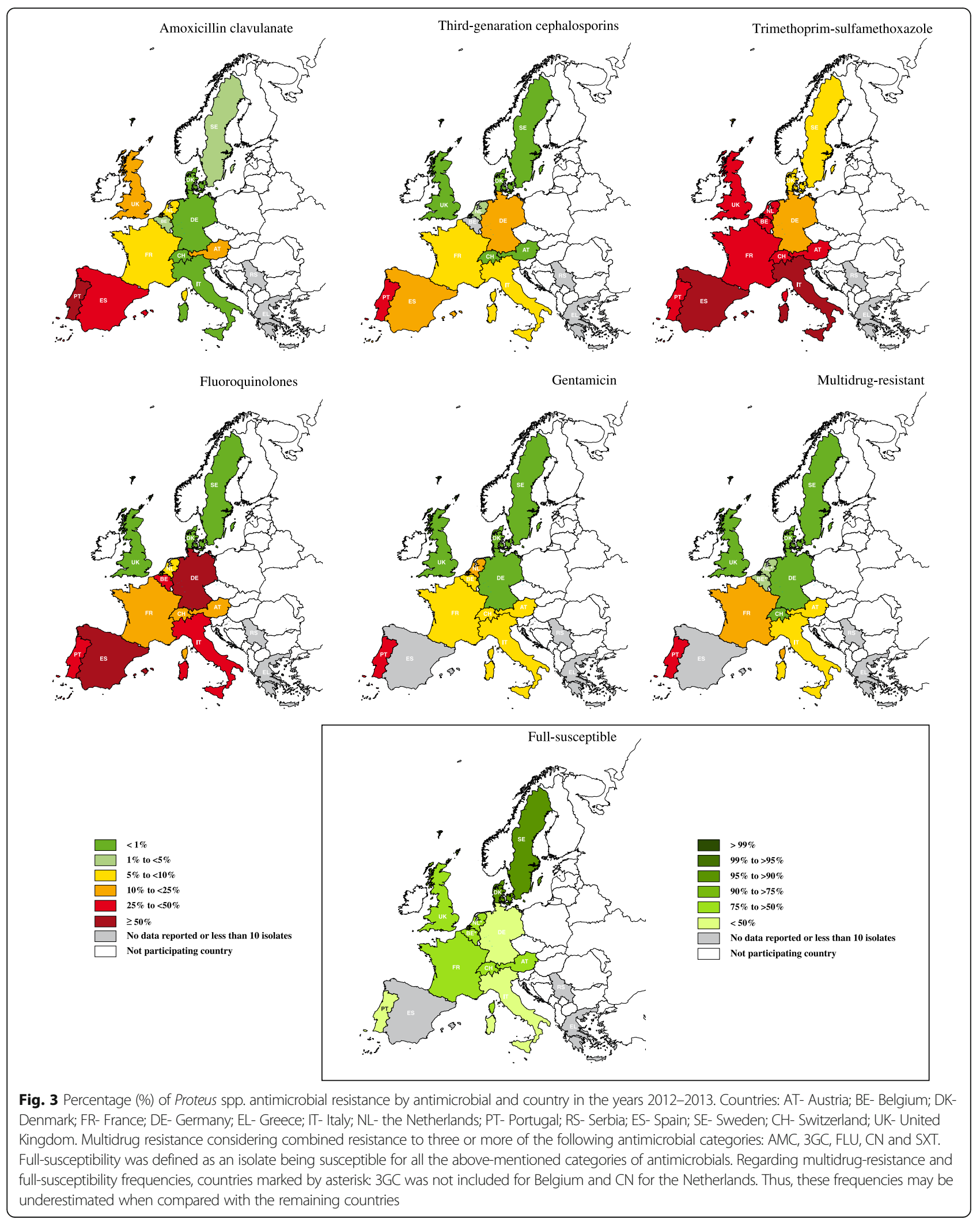


Table 5 Staphylococcus spp. and methicillin-resistance by country in 2012-2013

\begin{tabular}{|c|c|c|c|c|c|c|c|c|c|c|c|c|}
\hline \multirow{3}{*}{ Country $^{a}$} & \multicolumn{6}{|c|}{ Staphylococci species by country } & \multicolumn{6}{|c|}{ Methicillin-resistance within each group } \\
\hline & \multirow[t]{2}{*}{$\bar{N}$} & \multirow{2}{*}{$\begin{array}{l}\text { SA } \\
\% \\
(95 \% \mathrm{Cl})^{\mathrm{b}}\end{array}$} & \multirow{2}{*}{$\begin{array}{l}\mathrm{SP} \\
\% \\
(95 \% \mathrm{Cl})^{\mathrm{b}}\end{array}$} & \multirow{2}{*}{$\begin{array}{l}\text { CoPS } \\
\% \\
(95 \% \mathrm{Cl})^{b}\end{array}$} & \multirow{2}{*}{$\begin{array}{l}\text { CoNS } \\
\% \\
(95 \% \mathrm{Cl})^{b}\end{array}$} & \multirow{2}{*}{$\begin{array}{l}\text { SPP } \\
\% \\
(95 \% \mathrm{Cl})^{b}\end{array}$} & \multicolumn{2}{|l|}{$\overline{\mathrm{MRSA}}$} & \multicolumn{2}{|l|}{ MRSP } & \multicolumn{2}{|c|}{ MRCONS } \\
\hline & & & & & & & $\begin{array}{l}n \\
\text { tested }\end{array}$ & $\begin{array}{l}\text { R \% } \\
(95 \% \mathrm{Cl})^{\mathrm{b}}\end{array}$ & $\begin{array}{l}n \\
\text { tested }\end{array}$ & $\begin{array}{l}\text { R \% } \\
(95 \% \mathrm{Cl})^{\mathrm{b}}\end{array}$ & $\begin{array}{l}n \\
\text { tested }\end{array}$ & $\begin{array}{l}\mathrm{R} \% \\
(95 \% \mathrm{Cl})^{\mathrm{b}}\end{array}$ \\
\hline$\overline{\text { AT }}$ & 78 & $\begin{array}{l}7.69 \\
(1.78-13.61)\end{array}$ & $\begin{array}{l}19.23 \\
(10.48-27.98)\end{array}$ & $\begin{array}{l}6.41 \\
(0.97-11.85)\end{array}$ & $\begin{array}{l}56.41 \\
(45.41-67.41)\end{array}$ & $\begin{array}{l}10.26 \\
(3.52-16.99)\end{array}$ & 5 & $1 \mathrm{R} / 4 \mathrm{~S}$ & 15 & $\begin{array}{l}33.33 \\
(9.48-57.19)\end{array}$ & 43 & $\begin{array}{l}27.91 \\
(14.50-41.31)\end{array}$ \\
\hline $\mathrm{BE}$ & 122 & $\begin{array}{l}14.75 \\
(8.46-21.05)\end{array}$ & $\begin{array}{l}52.46 \\
(43.60-61.32)\end{array}$ & $\begin{array}{l}0 \\
-\end{array}$ & $\begin{array}{l}32.79 \\
(24.46-41.12)\end{array}$ & $\begin{array}{l}0 \\
-\end{array}$ & 18 & $\begin{array}{l}5.56 \\
(0-16.14)\end{array}$ & 64 & $\begin{array}{l}12.50 \\
(4.40-20.60)\end{array}$ & 40 & $\begin{array}{l}25.0 \\
(11.58-38.42)\end{array}$ \\
\hline DK & 52 & $\begin{array}{l}1.92 \\
(0-5.66)\end{array}$ & $\begin{array}{l}53.85 \\
(40.30-67.40)\end{array}$ & $\begin{array}{l}1.92 \\
(0-5.66)\end{array}$ & $\begin{array}{l}28.85 \\
(16.53-41.16)\end{array}$ & $\begin{array}{l}13.46 \\
(4.18-22.74)\end{array}$ & 1 & 15 & 27 & $\begin{array}{l}0 \\
-\end{array}$ & 15 & $\begin{array}{l}46.67 \\
(21.42-71.91)\end{array}$ \\
\hline FR & 242 & $\begin{array}{l}8.26 \\
(4.80-11.73)\end{array}$ & $\begin{array}{l}72.73 \\
(67.12-78.34)\end{array}$ & $\begin{array}{l}0 \\
-\end{array}$ & $\begin{array}{l}19.01 \\
(14.06-23.95)\end{array}$ & $\begin{array}{l}0 \\
-\end{array}$ & 20 & $\begin{array}{l}40.0 \\
(18.53-61.47)\end{array}$ & 0 & - & 46 & $\begin{array}{l}17.39 \\
(6.44-28.34)\end{array}$ \\
\hline $\mathrm{DE}$ & 64 & $\begin{array}{l}4.69 \\
(0-9.87)\end{array}$ & $\begin{array}{l}50.0 \\
(37.75-62.25)\end{array}$ & $\begin{array}{l}0 \\
-\end{array}$ & $\begin{array}{l}39.06 \\
(27.11-51.02)\end{array}$ & $\begin{array}{l}6.25 \\
(0.32-12.18)\end{array}$ & 0 & $\begin{array}{l}- \\
-\end{array}$ & 0 & $\begin{array}{l}- \\
-\end{array}$ & 0 & - \\
\hline EL & 10 & $\begin{array}{l}0 \\
-\end{array}$ & $\begin{array}{l}10.0 \\
(0-28.59)\end{array}$ & $\begin{array}{l}0 \\
-\end{array}$ & $\begin{array}{l}0 \\
-\end{array}$ & $\begin{array}{l}90 \\
(71.41-100)\end{array}$ & - & $\begin{array}{l}- \\
-\end{array}$ & 1 & $\begin{array}{l}1 \mathrm{R} \\
-\end{array}$ & - & $\begin{array}{l}- \\
-\end{array}$ \\
\hline $\mathrm{IT}$ & 19 & $\begin{array}{l}0 \\
-\end{array}$ & $\begin{array}{l}94.74 \\
(84.70-100)\end{array}$ & $\begin{array}{l}0 \\
-\end{array}$ & $\begin{array}{l}5.26 \\
(0-15.30)\end{array}$ & $\begin{array}{l}0 \\
-\end{array}$ & - & $\begin{array}{l}- \\
-\end{array}$ & 18 & $\begin{array}{l}50.0 \\
(26.90-73.10)\end{array}$ & 0 & $\begin{array}{l}- \\
-\end{array}$ \\
\hline $\mathrm{NL}$ & 365 & $\begin{array}{l}4.93 \\
(1.12-2.71)\end{array}$ & $\begin{array}{l}47.95 \\
(42.82-53.07)\end{array}$ & $\begin{array}{l}0 \\
-\end{array}$ & $\begin{array}{l}47.12 \\
(42.0-52.24)\end{array}$ & $\begin{array}{l}0 \\
-\end{array}$ & 0 & $\begin{array}{l}- \\
-\end{array}$ & 174 & $\begin{array}{l}10.92 \\
(6.29-15.55)\end{array}$ & 172 & $\begin{array}{l}0.58 \\
(0-1.72)\end{array}$ \\
\hline PT & 7 & $1 S A$ & $\begin{array}{l}4 \mathrm{SP} \\
-\end{array}$ & OCOPS & OCONS & $2 S P P$ & 1 & $\begin{array}{l}1 \mathrm{R} \\
-\end{array}$ & 4 & $2 \mathrm{R} / 2 \mathrm{~S}$ & 0 & - \\
\hline RS & 0 & $\begin{array}{l}- \\
-\end{array}$ & $\begin{array}{l}- \\
-\end{array}$ & $\begin{array}{l}- \\
-\end{array}$ & $\begin{array}{l}- \\
-\end{array}$ & - & - & $\begin{array}{l}- \\
-\end{array}$ & - & - & - & - \\
\hline ES & 13 & $\begin{array}{l}0 \\
-\end{array}$ & $\begin{array}{l}0 \\
-\end{array}$ & $\begin{array}{l}15.38 \\
(0-35.0)\end{array}$ & $\begin{array}{l}46.15 \\
(19.05-73.25)\end{array}$ & $\begin{array}{l}38.46 \\
(12.02-64.91)\end{array}$ & - & $\begin{array}{l}- \\
-\end{array}$ & - & - & 0 & - \\
\hline SE & 325 & $\begin{array}{l}8.62 \\
(5.56-11.67)\end{array}$ & $\begin{array}{l}53.54 \\
(48.12-58.96)\end{array}$ & $\begin{array}{l}2.46 \\
(0.78-4.15)\end{array}$ & $\begin{array}{l}32.31 \\
(27.22-37.39)\end{array}$ & $\begin{array}{l}3.08 \\
(1.20-4.95)\end{array}$ & 28 & $\begin{array}{l}0 \\
-\end{array}$ & 174 & $\begin{array}{l}1.15 \\
(0-2.73)\end{array}$ & 105 & $\begin{array}{l}4.76 \\
(0.69-8.84)\end{array}$ \\
\hline $\mathrm{CH}$ & 46 & $\begin{array}{l}4.35 \\
(0-10.24)\end{array}$ & $\begin{array}{l}52.17 \\
(37.74-66.61)\end{array}$ & $\begin{array}{l}4.35 \\
(0-10.24)\end{array}$ & $\begin{array}{l}34.78 \\
(21.02-48.55)\end{array}$ & $\begin{array}{l}4.35 \\
(0-10.24)\end{array}$ & 2 & $\mathrm{OR} / 2 \mathrm{~S}$ & 20 & $\begin{array}{l}10.00 \\
(0-23.15)\end{array}$ & 15 & $\begin{array}{l}66.67 \\
(42.81-90.52)\end{array}$ \\
\hline UK & 32 & $\begin{array}{l}12.50 \\
(1.04-23.96)\end{array}$ & $\begin{array}{l}53.13 \\
(35.84-70.41)\end{array}$ & $\begin{array}{l}6.25 \\
(0-14.64)\end{array}$ & $\begin{array}{l}18.75 \\
(5.23-32.27)\end{array}$ & $\begin{array}{l}9.38 \\
(0-19.47)\end{array}$ & 3 & $\begin{array}{l}1 \mathrm{R} / 2 \mathrm{~S} \\
-\end{array}$ & 12 & $\begin{array}{l}8.33 \\
(0-23.97)\end{array}$ & 3 & $\begin{array}{l}1 \mathrm{R} / 2 \mathrm{~S} \\
-\end{array}$ \\
\hline
\end{tabular}

Staphylococci identification varied according to the country. Some countries identified staphylococci to species level, others to genus level and others included data on the coagulase test. Thus, the staphylococci results were grouped as follows: 1. Staphylococcus aureus (SA); 2 . Staphylococcus pseudintermedius (SP); 3. coagulase positive staphylococci (CoPS), 4. coagulase negative staphylococci (CoNS) and 5. other staphylococci (SPP). Group 2 includes staphylococci identified only as CoPS or staphylococci species known to be coagulase positive other than SA and SP. Group 3 includes staphylococci identified only as CoNS or staphylococci species known to be coagulase negative. Group 4 includes staphylococci identified as Staphylococcus spp

MRSA methicillin-resistant Staphylococcus aureus, MRSP methicillin-resistant Staphylococcus pseudintermedius, MRCoNS methicillin-resistant coagulase negative staphylococci

${ }^{2}$ Countries: AT, Austria; BE, Belgium; DK, Denmark; FR, France; DE, Germany; EL, Greece; IT, Italy; NL, the Netherlands; PT, Portugal; RS, Serbia; ES, Spain; SE, Sweden; $\mathrm{CH}$, Switzerland; UK, United Kingdom

${ }^{b} 95 \% \mathrm{Cl}, 95 \%$ Confidence interval

$N$, Total number of staphylococci

$n$ tested, number of staphylococci tested for methicillin-resistance within each group

isolated followed by coagulase negative staphylococci (CoNS) (Table 5). In general, the overall antimicrobial resistance levels in Southern countries were higher than in Northern countries (Fig. 4) as seen in Gram-negative bacteria (Figs. 2 and 3).

Besides the limited number of staphylococci, methicillinresistance results were also limited due to the identification only to genus level or lack of testing the appropriate antimicrobial surrogate. Denmark and Sweden showed the lowest S. pseudintermedius methicillin-resistance (MRSP) ( 0 and $1.15 \%$, respectively). The remaining countries had MRSP frequencies higher than $8 \%$, attaining $50 \%$ in Italy. Methicillin-resistance was also high in CoNS (Table 5).
Staphylococci SXT resistance ranged from 2.77 to $63.16 \%$ and showed similar geographical distribution to Gram-negative bacteria (Fig. 4). Among the participating countries, Staphylococci FLU resistance frequencies were higher in Italy $(42.11 \%)$ and again lower in Sweden $(1.54 \%)$ and Denmark (1.96\%), with the remaining countries varying between 6.59 and $26.92 \%$ (Table 6). Italy, Austria, Germany and Switzerland $\mathrm{CN}$ resistant staphylococci frequencies ranged between 26.32 and $9.76 \%$ while the remaining countries had less than $6 \%$ (Table 6).

Regarding resistance temporal trends, most countries had no significant changes in $E$. coli resistance over the 
Table 6 Percentage of resistance in Staphylococcus spp. by antimicrobial and country in 2012-2013

\begin{tabular}{|c|c|c|c|c|c|c|}
\hline \multirow[t]{2}{*}{ Country $^{a}$} & \multicolumn{2}{|l|}{$\underline{F L U}$} & \multicolumn{2}{|l|}{$\mathrm{CN}$} & \multicolumn{2}{|l|}{ SXT } \\
\hline & $n$ & $\begin{array}{l}\text { \% R }(95 \% \text { Cl) } \\
\text { [Stat. Dif.] }{ }^{\mathrm{C}}\end{array}$ & $n$ & $\begin{array}{l}\% \text { R }(95 \% \text { Cl) } \\
\text { [Stat. Dif.] }^{\mathrm{c}}\end{array}$ & $\bar{n}$ & $\begin{array}{l}\text { \% R }(95 \% \mathrm{Cl})^{\mathrm{b}} \\
\text { [Stat. Dif.] }^{\mathrm{C}}\end{array}$ \\
\hline$\overline{\mathrm{AT}}$ & 78 & $\begin{array}{l}26.92 \\
(17.08-36.77) \\
{[a, b]}\end{array}$ & 78 & $\begin{array}{l}19.23 \\
(10.48-27.98) \\
{[a]}\end{array}$ & 78 & $\begin{array}{l}20.51 \\
(11.55-29.47) \\
{[a, b]}\end{array}$ \\
\hline $\mathrm{BE}$ & 116 & $\begin{array}{l}7.76 \\
(2.89-12.63) \\
{[c, d]}\end{array}$ & 107 & $\begin{array}{l}3.74 \\
(0.14-7.33) \\
{[b]}\end{array}$ & 122 & $\begin{array}{l}13.11 \\
(7.12-19.10) \\
{[a, b, c]}\end{array}$ \\
\hline DK & 51 & $\begin{array}{l}1.96 \\
(0.00-5.77) \\
{[c, e, f]}\end{array}$ & 51 & $\begin{array}{l}3.92 \\
(0.00-9.25) \\
{[b]}\end{array}$ & 51 & $\begin{array}{l}0 \\
- \\
{[d]}\end{array}$ \\
\hline$F R$ & 238 & $\begin{array}{l}23.53 \\
(18.14-28.92) \\
{[a, g]}\end{array}$ & 237 & $\begin{array}{l}5.06 \\
(2.27-7.85) \\
{[b]}\end{array}$ & 242 & $\begin{array}{l}11.57 \\
(7.54-15.60) \\
{[a, c]}\end{array}$ \\
\hline $\mathrm{DE}$ & 55 & $\begin{array}{l}18.18 \\
(7.99-28.38) \\
{[a, d, g]}\end{array}$ & 55 & $\begin{array}{l}10.91 \\
(2.67-19.15) \\
{[a, b]}\end{array}$ & 55 & $\begin{array}{l}23.64 \\
(12.41-34.86) \\
{[b]}\end{array}$ \\
\hline $\mathrm{EL}$ & 10 & $\begin{array}{l}20.00 \\
(0.00-44.79) \\
{[a, d, e, g N h]}\end{array}$ & 0 & $\begin{array}{l}- \\
- \\
-\end{array}$ & 9 & $\begin{array}{l}1 \mathrm{R} / 8 \mathrm{~S} \\
- \\
-\end{array}$ \\
\hline IT & 19 & $\begin{array}{l}42.11 \\
(19.90-64.31) \\
{[a]}\end{array}$ & 19 & $\begin{array}{l}26.32 \\
(6.52-46.12) \\
{[a]}\end{array}$ & 19 & $\begin{array}{l}63.16 \\
(41.47-84.85) \\
{[\mathrm{e}]}\end{array}$ \\
\hline $\mathrm{NL}$ & 364 & $\begin{array}{l}6.59 \\
(4.04-9.14) \\
{[C, i]}\end{array}$ & 9 & $\begin{array}{l}1 \mathrm{R} / 8 \mathrm{~S} \\
- \\
-\end{array}$ & 365 & $\begin{array}{l}11.51 \\
(8.23-14.78) \\
{[c]}\end{array}$ \\
\hline PT & 6 & $\begin{array}{l}3 R / 3 S \\
- \\
-\end{array}$ & 7 & $\begin{array}{l}2 \mathrm{R} / 5 \mathrm{~S} \\
- \\
-\end{array}$ & 7 & $\begin{array}{l}1 \mathrm{R} / 6 \mathrm{~S} \\
- \\
-\end{array}$ \\
\hline RS & 0 & $\begin{array}{l}- \\
- \\
-\end{array}$ & 0 & $\begin{array}{l}- \\
- \\
-\end{array}$ & 0 & $\begin{array}{l}- \\
-\end{array}$ \\
\hline ES & 13 & $\begin{array}{l}15.38 \\
(0.00-35.00) \\
{[a, d, e, h, i]}\end{array}$ & 8 & $\begin{array}{l}0 \mathrm{R} / 8 \mathrm{~S} \\
- \\
-\end{array}$ & 11 & $\begin{array}{l}18.18 \\
(0.00-40.97) \\
{[a, b, c]}\end{array}$ \\
\hline SE & 325 & $\begin{array}{l}1.54 \\
(0.20-2.88) \\
{[f]}\end{array}$ & 325 & $\begin{array}{l}0 \\
- \\
{[c]}\end{array}$ & 325 & $\begin{array}{l}2.77 \\
(0.99-4.55) \\
{[d]}\end{array}$ \\
\hline $\mathrm{CH}$ & 41 & $\begin{array}{l}24.39 \\
(11.25-37.54) \\
{[a, b]}\end{array}$ & 41 & $\begin{array}{l}9.76 \\
(0.67-18.84) \\
{[a, b]}\end{array}$ & 41 & $\begin{array}{l}19.51 \\
(7.38-31.64) \\
{[a, b, c]}\end{array}$ \\
\hline UK & 31 & $\begin{array}{l}12.90 \\
(1.10-24.70) \\
{[b, c, e, g]}\end{array}$ & 22 & $\begin{array}{l}4.55 \\
(0.00-13.25) \\
{[a, b, c]}\end{array}$ & 30 & $\begin{array}{l}16.67 \\
(3.33-30.00) \\
{[a, b, c]}\end{array}$ \\
\hline
\end{tabular}

FLU fluoroquinolones, CN gentamicin, SXT trimethoprim/sulfamethoxazole

${ }^{a}$ Countries: AT, Austria; BE, Belgium; DK, Denmark; FR, France; DE, Germany; EL, Greece; IT, Italy; NL, the Netherlands; PT, Portugal; RS, Serbia; ES, Spain; SE, Sweden; $\mathrm{CH}$, Switzerland; UK, United Kingdom

${ }^{\mathrm{b}} 95 \% \mathrm{Cl}, 95 \%$ Confidence interval

'Stat. Dif., Statistical significant differences. Countries with no statistical difference are marked with the same letter. Countries were compared by fisher exact test with an alpha value of 0.05 . Countries with less than ten tested isolates were not compared

$n$, Total number of Staphylococcus spp. tested for the considered antimicrobial category

time periods considered (Table 7). Belgium showed a significant decrease in E. coli resistance to all antimicrobials and an increase in full susceptible isolates. Denmark (AMC, FLU, SXT), France (3GC, FLU), the Netherlands (3GC, FLU, SXT, MDR) and Sweden (CN,
MDR) had also significant decreases in E. coli resistance over time (Table 7). However, the Netherlands (AMC) and Switzerland $(\mathrm{CN})$ had a significant increase in E. coli resistance (Table 7). A rising trend was also detected in Proteus spp. FLU resistance from Belgium (Table 8). 


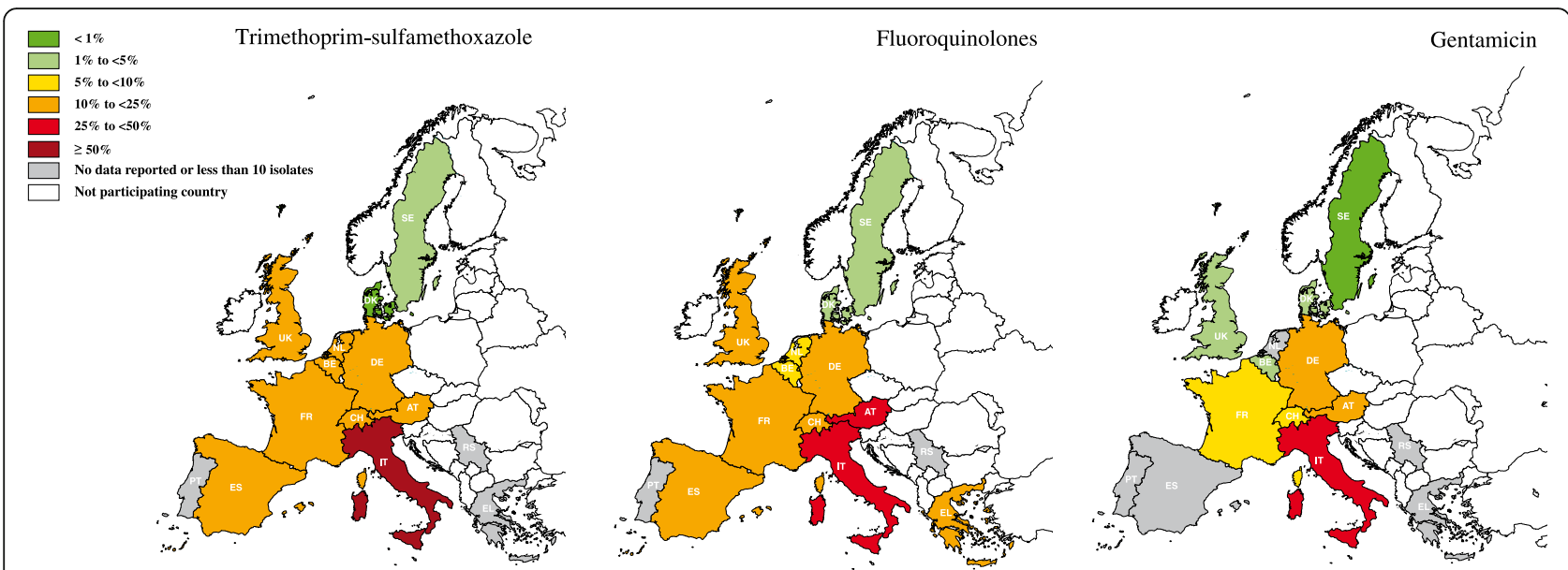

Fig. 4 Percentage (\%) of Staphylococcus spp. antimicrobial resistance by antimicrobial and country in the years 2012-2013. Countries: AT- Austria; BE- Belgium; DK- Denmark; FR- France; DE- Germany; EL- Greece; IT- Italy; NL- the Netherlands; PT- Portugal; RS- Serbia; ES- Spain; SE- Sweden; $\mathrm{CH}$ - Switzerland; UK- United Kingdom

\section{Discussion}

Published data on antimicrobial resistance in bacteria isolated from companion animal UTIs over Europe is scarce [19] and the comparison between studies is impaired by the use of different inclusion criteria and different time periods. Moreover, UTI resistance frequencies are usually reported together with susceptibility data from other sites of infection [11, 28], combining different bacteria genera $[8,9]$ and several countries $[29,30]$. These facts impair the establishment of a global epidemiological overview of UTI bacteria resistance in Europe. This is the first large study to analyse antimicrobial susceptibility data of canine and feline isolates from several European countries allowing an epidemiological overview of UTI resistance trends in Europe.

In accordance to previous studies [7-9, 31, 32], E. coli was the most frequently isolated bacteria in dogs and cats. Enterococcus presented a significantly higher frequency in cats and Proteus spp. in dogs. While not compared in previous studies, this difference could be expected based on some published data focused on cats $[7,9]$ and dogs [32] separately.

One of the most important findings from this study was the overall higher resistance frequencies found in the Southern countries (Italy, Greece, Portugal and Spain) when compared with the Northern countries (Denmark and Sweden). The lower frequency of antimicrobial resistance in Northern countries, such as Sweden, is likely a consequence of the tight regulations and surveillance on antimicrobial prescribing and resistance in companion animals. In light of the present results, such strategies could be useful in aiming the reduction of antimicrobial resistance in the Southern countries.

\section{Resistance to Beta-Lactams Amoxicillin-clavulanate}

Considering that AMC is one of the most used antimicrobials in animals, the levels of resistance detected in this study are worrisome, especially in the Southern countries. Previous published reports showed different frequencies of AMC resistance in E. coli and in Proteus spp. that are likely due to the fact they report to different time frames and inclusion criteria [10,32-37]. In the absence of clinical data it is not possible to know if this resistance relates to uncomplicated or complicated UTI [19]. Thus, these results need to be further investigated in order to establish whether AMC is a suitable empiric therapeutic choice for companion animals UTI in Southern Europe.

\section{Third generation cephalosporins}

Southern countries had also higher levels of resistance to 3GCs. Although Greece was not included due to limited data, considering that seven out of the nine tested isolates were resistant to $3 \mathrm{GCs}$, one can expect the prevalence of 3GC resistance to be high. Previous studies in Portugal found a considerable lower $3 \mathrm{GC}$ resistance value (1.4\%) in $E$. coli from dogs in earlier years [10]. In the present work, the lower Swedish results for 3GC resistance in $E$. coli and Proteus spp. are in agreement with early studies [38]. Being of critical importance to humans [39], prudent use of $3 \mathrm{GC}$ is of upmost importance.

\section{Methicillin-resistance}

The frequency of methicillin-resistant staphylococci, especially S. pseudintermedius and CoNS, varied considerably between countries and confirmed previous reports on a low MRSP prevalence in Scandinavia compared to elsewhere in Europe [40]. Resistance to methicillin in 
Table 7 Temporal trends of antimicrobial resistance in Escherichia coli by country

\begin{tabular}{|c|c|c|c|c|c|c|c|}
\hline \multirow{2}{*}{$\begin{array}{l}\text { Country }^{a} \\
\text { (Years) }\end{array}$} & AMC & $3 G C$ & FLU & $\mathrm{CN}$ & SXT & MDR & Fulls \\
\hline & $\begin{array}{l}\mathrm{OR}^{\mathrm{b}} \\
(95 \% \mathrm{Cl})^{\mathrm{c}} \\
P \text { value }\end{array}$ & $\begin{array}{l}\mathrm{OR}^{\mathrm{b}} \\
(95 \% \mathrm{Cl})^{\mathrm{c}} \\
P \text { value }\end{array}$ & $\begin{array}{l}\mathrm{OR}^{\mathrm{b}} \\
(95 \% \mathrm{Cl})^{c} \\
P \text { value }\end{array}$ & $\begin{array}{l}\mathrm{OR}^{\mathrm{b}} \\
(95 \% \mathrm{Cl})^{c} \\
P \text { value }\end{array}$ & $\begin{array}{l}\mathrm{OR}^{\mathrm{b}} \\
(95 \% \mathrm{Cl})^{c} \\
P \text { value }\end{array}$ & $\begin{array}{l}\mathrm{OR}^{\mathrm{b}} \\
(95 \% \mathrm{Cl})^{c} \\
P \text { value }\end{array}$ & $\begin{array}{l}\mathrm{OR}^{\mathrm{b}} \\
(95 \% \mathrm{Cl})^{\mathrm{c}} \\
P \text { value }\end{array}$ \\
\hline $\begin{array}{l}\mathrm{BE} \\
(2010-13)\end{array}$ & $\begin{array}{l}0.787 \\
(0.646-0.960) \\
0.0180\end{array}$ & - & $\begin{array}{l}0.749 \\
(0.635-0.882) \\
0.0006\end{array}$ & $\begin{array}{l}0.677 \\
(0.507-0.904) \\
0.0081\end{array}$ & $\begin{array}{l}0.796 \\
(0.695-0.912) \\
0.0010\end{array}$ & $\begin{array}{l}0.529^{d} \\
(0.393-0.712) \\
<0.0001\end{array}$ & $\begin{array}{l}1.275^{\mathrm{d}} \\
(1.127-1.442) \\
0.0001\end{array}$ \\
\hline $\begin{array}{l}\text { DK } \\
\text { (2008-13) }\end{array}$ & $\begin{array}{l}0.698 \\
(0.500-0.976) \\
0.0357\end{array}$ & $\begin{array}{l}0.869 \\
(0.646-1.169) \\
0.3533\end{array}$ & $\begin{array}{l}0.742 \\
(0.565-0.976) \\
0.0325\end{array}$ & $\begin{array}{l}0.926 \\
(0.620-1.384) \\
0.7086\end{array}$ & $\begin{array}{l}0.793 \\
(0.642-0.980) \\
0.0316\end{array}$ & $\begin{array}{l}0.874 \\
(0.615-1.242) \\
0.4528\end{array}$ & $\begin{array}{l}1.396 \\
(1.156-1.684) \\
0.0005\end{array}$ \\
\hline $\begin{array}{l}\text { FR } \\
\text { (2010-13) }\end{array}$ & $\begin{array}{l}0.885 \\
(0.780-1.005) \\
0.0606\end{array}$ & $\begin{array}{l}0.859 \\
(0.749-0.987) \\
0.0314\end{array}$ & $\begin{array}{l}0.822 \\
(0.727-0.928) \\
0.0016\end{array}$ & $\begin{array}{l}0.938 \\
(0.734-1.200) \\
0.6121\end{array}$ & $\begin{array}{l}0.960 \\
(0.853-1.080) \\
0.4997\end{array}$ & $\begin{array}{l}0.901 \\
(0.782-1.037) \\
0.1448\end{array}$ & $\begin{array}{l}1.112 \\
(1.002-1.233) \\
0.0456\end{array}$ \\
\hline $\begin{array}{l}\text { DE } \\
\text { (2009-13) }\end{array}$ & $\begin{array}{l}1.029 \\
(0.779-1.358) \\
0.8424\end{array}$ & $\begin{array}{l}1.076 \\
(0.805-1.438) \\
0.6211\end{array}$ & $\begin{array}{l}1.185 \\
(0.912-1.540) \\
0.2037\end{array}$ & $\begin{array}{l}0.856 \\
(0.520-1.409) \\
0.5397\end{array}$ & $\begin{array}{l}1.040 \\
(0.831-1.302) \\
0.7317\end{array}$ & $\begin{array}{l}1.111 \\
(0.801-1.541) \\
0.5295\end{array}$ & $\begin{array}{l}0.941 \\
(0.780-1.136) \\
0.5281\end{array}$ \\
\hline $\begin{array}{l}\mathrm{EL}^{\mathrm{e}} \\
(2009-13)\end{array}$ & $\begin{array}{l}1.534 \\
(0.851-2.766) \\
0.1545\end{array}$ & $\begin{array}{l}1.083 \\
(0.586-2.003) \\
0.7992\end{array}$ & $\begin{array}{l}0.924 \\
(0.630-1.355) \\
0.6855\end{array}$ & $\begin{array}{l}- \\
- \\
-\end{array}$ & $\begin{array}{l}0.880 \\
(0.596-1.301) \\
0.5229\end{array}$ & $\begin{array}{l}- \\
- \\
-\end{array}$ & $\begin{array}{l}- \\
- \\
-\end{array}$ \\
\hline $\begin{array}{l}\text { IT } \\
\text { (2009-2013) }\end{array}$ & $\begin{array}{l}1.175 \\
(0.844-1.637 \\
0.3391)\end{array}$ & $\begin{array}{l}1.017 \\
(0.749-1.383) \\
0.9127\end{array}$ & $\begin{array}{l}0.828 \\
(0.629-1.090) \\
0.1784\end{array}$ & $\begin{array}{l}1.007 \\
(0.700-1.449) \\
0.9686\end{array}$ & $\begin{array}{l}0.769 \\
(0.582-1.016) \\
0.0645\end{array}$ & $\begin{array}{l}1.065 \\
(0.761-1.490) \\
0.7147\end{array}$ & $\begin{array}{l}1.248 \\
(0.953-1.634) \\
0.1076\end{array}$ \\
\hline $\begin{array}{l}\mathrm{NL} \\
(2008-13)\end{array}$ & $\begin{array}{l}1.108 \\
(1.026-1.197) \\
0.0088\end{array}$ & $\begin{array}{l}0.465 \\
(0.402-0.539) \\
<0.0001\end{array}$ & $\begin{array}{l}0.916 \\
(0.841-0.999) \\
0.0464\end{array}$ & $\begin{array}{l}0.682 \\
(0.327-1.422) \\
0.3071\end{array}$ & $\begin{array}{l}0.917 \\
(0.859-0.978) \\
0.0083\end{array}$ & $\begin{array}{l}0.380^{d} \\
(0.320-0.450) \\
<0.0001\end{array}$ & $\begin{array}{l}1.648^{d} \\
(1494 .-1.818) \\
<0.0001\end{array}$ \\
\hline $\begin{array}{l}\text { PT } \\
\text { (2008-13) }\end{array}$ & $\begin{array}{l}1.139 \\
(0.913-1.419) \\
0.2482\end{array}$ & $\begin{array}{l}1.187 \\
(0.945-1.492) \\
0.1411\end{array}$ & $\begin{array}{l}1.029 \\
(0.823-1.287) \\
0.8029\end{array}$ & $\begin{array}{l}1.222 \\
(0.899-1.660) \\
0.2010\end{array}$ & $\begin{array}{l}1.087 \\
(0.867-1.364) \\
0.4680\end{array}$ & $\begin{array}{l}1.156 \\
(0.898-1.488) \\
0.2601\end{array}$ & $\begin{array}{l}0.797 \\
(0.629-1.010) \\
0.0608\end{array}$ \\
\hline $\begin{array}{l}\text { ES } \\
(2010-13)\end{array}$ & $\begin{array}{l}1.372 \\
(0.855-2.201) \\
0.1899\end{array}$ & $\begin{array}{l}1.551 \\
(0.857-2.808) \\
0.1474\end{array}$ & $\begin{array}{l}0.801 \\
(0.529-1214) \\
0.2965\end{array}$ & $\begin{array}{l}0.859 \\
(0.467-1.578) \\
0.6238\end{array}$ & $\begin{array}{l}0.752 \\
(0.489-1.156) \\
0.1939\end{array}$ & $\begin{array}{l}1.237 \\
(0.677-2.258) \\
0.4897\end{array}$ & $\begin{array}{l}0.944 \\
(0.570-1.564) \\
0.8234\end{array}$ \\
\hline $\begin{array}{l}\text { SE } \\
(2008-13)\end{array}$ & $\begin{array}{l}0.976 \\
(0.915-1.041) \\
0.4569\end{array}$ & $\begin{array}{l}- \\
-\end{array}$ & $\begin{array}{l}0.980 \\
(0.827-1.147) \\
0.8018\end{array}$ & $\begin{array}{l}0.700 \\
(0.562-0.872) \\
0.0015\end{array}$ & $\begin{array}{l}0.961 \\
(0.892-1.037) \\
0.3059\end{array}$ & $\begin{array}{l}0.697 \\
(0.493-0.985) \\
0.0407\end{array}$ & $\begin{array}{l}1.035 \\
(0.965-1.110) \\
0.3341\end{array}$ \\
\hline $\begin{array}{l}\mathrm{CH} \\
(2008-13)\end{array}$ & $\begin{array}{l}1.143 \\
(0.905-1.445) \\
0.2621\end{array}$ & $\begin{array}{l}1.116 \\
(0.861-1.447) \\
0.4067\end{array}$ & $\begin{array}{l}1.007 \\
(0.841-1.205) \\
0.9426\end{array}$ & $\begin{array}{l}1.493 \\
(1.009-2.208) \\
0.0451\end{array}$ & $\begin{array}{l}1.080 \\
(0.901-1.294) \\
0.4050\end{array}$ & $\begin{array}{l}1.189 \\
(0.920-1.536) \\
0.1863\end{array}$ & $\begin{array}{l}1.025 \\
(0.877-1.197) \\
0.7594\end{array}$ \\
\hline $\begin{array}{l}\text { UK } \\
(2008-13)\end{array}$ & $\begin{array}{l}1.075 \\
(0.857-1.357) \\
0.5194\end{array}$ & $\begin{array}{l}1.106 \\
(0.873-1.400) \\
0.4041\end{array}$ & $\begin{array}{l}0.945 \\
(0.739-1.208) \\
0.6511\end{array}$ & $\begin{array}{l}1.306 \\
(0.792-2.155) \\
0.2952\end{array}$ & $\begin{array}{l}0.972 \\
(0.797-1.185) \\
0.7778\end{array}$ & $\begin{array}{l}1.355 \\
(0.954-1.925) \\
0.0892\end{array}$ & $\begin{array}{l}1.154 \\
(0.950-1.401) \\
0.1492\end{array}$ \\
\hline
\end{tabular}

AMC amoxicillin clavulanate, 3GC third generation cephalosporins, FLU fluoroquinolones, CN gentamicin, SXT trimethoprim/sulfamethoxazole, MDR multidrugresistant, Fulls fully-susceptible

${ }^{a} B E$, Belgium; DK, Denmark; FR, France; DE, Germany; EL, Greece; IT, Italy; NL, the Netherlands; PT, Portugal; ES, Spain; SE, Sweden; CH, Switzerland; UK, United Kingdom

${ }^{\mathrm{b}} \mathrm{OR}$, Odds ratio

${ }^{\mathrm{c}} 95 \% \mathrm{Cl}, 95 \%$ Confidence interval

${ }^{\mathrm{d}} \mathrm{MDR}$ and FullS temporal trends do not include resistance to $3 \mathrm{GC}$ for Belgium and CN for the Netherlands

e Data regarding the years 2010 and 2012 were excluded from Greece resistance trends analysis since less than ten isolates were tested in those years

Statistically significant trends are highlighted in bold

coagulase-positive Staphylococcus (S. aureus and S. pseudintermedius) was detected in this study and is a great animal and public health concern [41]. Currently, the recommended methods for the detection of methicillinresistance in Staphylococci are manly phenotypic but in some circumstances the molecular detection of the $m e c A$ gene is clinically and epidemiologically necessary [22, 23, 42]. This should be taken in consideration for the harmonization of veterinary susceptibility testing in Europe.

\section{Resistance to fluoroquinolones}

In this study, high resistance frequencies towards the fluoroquinolones were found in E. coli, Proteus spp and Staphylococcus spp. isolates in the southern European countries but also in Proteus spp. from Germany, Belgium and Switzerland and Staphylococcus spp. from Austria, Switzerland and France. Several authors [32, 34, $43,44]$ have reported lower FLU resistance frequencies than the ones found in this study, especially regarding the Southern countries [43, 44]. The results of high 
Table 8 Temporal trends of antimicrobial resistance in Proteus spp. by country

\begin{tabular}{|c|c|c|c|}
\hline \multirow{2}{*}{$\begin{array}{l}\text { Country }^{a} \\
\text { (Years) }\end{array}$} & AMC & FLU & SXT \\
\hline & $\begin{array}{l}\mathrm{OR}^{\mathrm{b}}(95 \% \mathrm{Cl})^{\mathrm{c}} \\
P \text { value }\end{array}$ & $\begin{array}{l}\mathrm{OR}^{\mathrm{b}}(95 \% \mathrm{Cl})^{\mathrm{c}} \\
P \text { value }\end{array}$ & $\begin{array}{l}\mathrm{OR}^{\mathrm{b}}(95 \% \mathrm{Cl})^{\mathrm{c}} \\
P \text { value }\end{array}$ \\
\hline$\overline{B E}(2010-13)$ & $\begin{array}{l}0.627(0.312-1.259) \\
0.1889\end{array}$ & $\begin{array}{l}1.292(1.006-1.659) \\
0.0450\end{array}$ & $\begin{array}{l}0.891(0.726-1.092) \\
0.2649\end{array}$ \\
\hline FR (2010-13) & $\begin{array}{l}0.945(0.625-1.431) \\
0.7908\end{array}$ & $\begin{array}{l}1.004(0.764-1.319) \\
0.9770\end{array}$ & $\begin{array}{l}0.954(0.756-1.205) \\
0.6944\end{array}$ \\
\hline NL (2008-13) & $\begin{array}{l}1.092(0.860-1.387) \\
0.4709\end{array}$ & $\begin{array}{l}0.970(0.829-1.135) \\
0.7067\end{array}$ & $\begin{array}{l}1.010(0.903-1.129) \\
0.8660\end{array}$ \\
\hline SE (2008-13) & $\begin{array}{l}0.892(0.657-1.210) \\
0.4620\end{array}$ & $\begin{array}{l}0.884(0.494-1.583) \\
0.6788\end{array}$ & $\begin{array}{l}0.941(0.759-1.165) \\
0.5756\end{array}$ \\
\hline
\end{tabular}

AMC amoxicillin clavulanate, FLU fluoroquinolones, SXT trimethoprim/sulfamethoxazole

Statistically significant trends are highlighted in bold

${ }^{a} \mathrm{BE}$, Belgium; DK, Denmark; FR, France; NL, the Netherlands; SE, Sweden; CH, Switzerland

${ }^{\mathrm{b}} \mathrm{OR}$, Odds ratio

${ }^{\mathrm{c}} 95 \% \mathrm{Cl}, 95 \%$ Confidence interval

resistance frequencies towards the fluoroquinolones found in this study are concerning because fluoroquinolones are considered a good first choice for pyelonephritis treatment and should otherwise be used as a second line antimicrobial [19].

\section{Resistance to folate inhibitors and to aminoglicosides}

In this study, resistance to SXT in Europe was high, especially in E. coli and Proteus spp.. The higher SXT resistance found in Proteus spp., than in E. coli from several European countries, is consistent with other reports [34, 36, 45]. Compared with previous studies, these results show a superior SXT resistance in E. coli and Proteus spp. from Italy and Portugal [10, 43] and Staphylococcus spp. from Belgium [36].

Also in agreement to previous studies, gentamicin was the antimicrobial with lower resistance in E. coli, Proteus spp. and Staphylococcus spp. all-over Europe [32, 34, 35, 37, 38, 43, 45]. Nevertheless, the distribution seemed to follow the same pattern, with increased resistance in Southern over Northern countries.

\section{Multidrug-resistance}

Finally, MDR bacteria presented the worst scenario once again in E. coli from Southern countries and in Proteus spp. from Portugal. The emergence of MDR bacteria in companion animals has been previously described $[46,47]$ and represents a great therapeutic challenge and public health concern. However, MDR/FullS frequencies are seldom reported and published data account for different antimicrobials, thus impairing any comparisons with the present results [10, 32, 35, 38, 45].

\section{Trends in antimicrobial resistance}

The surveillance of antimicrobial resistance is an important tool to guide the implementation of antimicrobial stewardship strategies. In this study, most countries had no significant changes in antimicrobial resistance over the time frame considered. Nevertheless, decreasing trends in antimicrobial resistance were found in E. coli. These encouraging trends were not detected in AMC and $\mathrm{CN}$ resistance in E. coli from the Netherlands and Switzerland, respectively, where an increasing trend was observed. Although no changes over time were detected in E. coli resistance against AMC and 3CGs in Portugal, the considerably lower resistance frequencies previously reported in earlier years [10], point to a possible increasing trend [33]. The same may be the case for E. coli AMC resistance in Germany and Switzerland [34, 35].

Despite reporting clear trends such as the difference in resistance between Northern and Southern countries, data from this study should be interpreted with caution. Due to the retrospective nature of this study, data on clinical history such as the type of UTI and previous antimicrobial treatment were unavailable. Furthermore, the use of laboratory data may represent a bias towards resistance, since urine cultures from complicated cases tend to be requested more often than simple uncomplicated UTI $[8,31]$. These limitations are not restricted to certain countries, and are therefore not likely to hamper comparison of data across borders. Given the limitations of retrospective studies, a veterinary European surveillance network gathering data prospectively on antimicrobial resistance, as well as, on clinical data is of the upmost importance to facilitate development of national evidencebased guidelines that take into consideration type of UTI, local regulations and patterns of antimicrobial resistance.

The use of different susceptibility testing methods and different clinical breakpoints is considered a major limitation. The lack of harmonization became evident in this study when trying to compare 3GC and methicillinresistance. Although it also happens in the well-established 
EARS network reports of resistance on bacteria from human invasive infections [18], this limitation weakens the comparison of resistance between countries in the present and future surveillance studies. This harmonization would allow future within and between countries resistance frequencies comparisons over time and would also provide relevant information on the impact of different antimicrobial usage policies. Thus, the authors agree that the harmonization of methods and interpretative criteria in veterinary medicine should be a priority. The role of the new veterinary committee on antimicrobial susceptibility testing VetCAST [48] may be crucial in this harmonization process. Despite these limitations, the results from this study provide relevant and updated information on the current antimicrobial resistance in UTI bacteria from companion animals in Europe. Similar studies should also be conducted regarding other types of infection to improve the awareness on the European distribution of antimicrobial resistance in companion animals. Ideally, monitoring of companion animal antimicrobial resistance should be implemented in Europe, as it is the case for food producing animals. Such surveillance would provide crucial information to promote the appropriate use of antimicrobial and therefore limit the spread of resistance.

\section{Conclusions}

This work brings new insights into the current scenario of the European antimicrobial resistance bacteria isolated from companion animals with UTI. An important finding from this study was the higher frequency of resistance in Southern European countries (Italy, Greece, Spain, Portugal) when compared to Northern European countries (Denmark, Sweden). Furthermore, there is an evident need to harmonize methods and interpretative criteria in veterinary medicine. Given the limitations of retrospective studies, an European surveillance network gathering data on antimicrobial resistance is of the upmost importance to facilitate the development of national evidence-based guidelines.

\footnotetext{
Abbreviations

3GC: Third generation cephalosporins; $95 \%$ Cl: $95 \%$ confidence interval; AMC: Amoxicillin-clavulanate; AMP: Ampicillin; AT: Austria; BE: Belgium; CAZ: Ceftazidime; CH: Switzerland; CIP: Ciprofloxacin; CLSI: Clinical and Laboratory Standards Institute; CN: Gentamicin; CN120: Gentamicin highlevel; CoNS: Coagulase negative staphylococci; CoPS: Coagulase positive staphylococci; CPD: Cefpodoxime; CTX: Cefotaxime; CVN: Cefovecin; DE: Germany; DK: Denmark; EARS-Net: European Antimicrobial Resistance Surveillance Network; EFT: Ceftiofur; EL: Greece; ENR: Enrofloxacin; ES: Spain; FLU: Fluoroquinolones; FOX: Cefoxitin; FR: France; FullS: Full-susceptibility; IT: Italy; MAR: Marbofloxacin; MDR: Multidrug-resistant; MRCoNS: Methicillinresistant coagulase negative staphylococci; MRSA: Methicillin-resistant Staphylococcus aureus; MRSP: Methicillin-resistant Staphylococcus pseudintermedius; NL: the Netherlands; OR: Odds ratio; OX: Oxacillin; P: Penicillin; PT: Portugal; R: Full resistant; RS: Serbia; S: Susceptible; SA: Staphylococcus aureus; SE: Sweden; SP: Staphylococcus pseudintermedius; Stat. Dif.: Statistical significant differences; SXT: Trimethoprim/ sulfamethoxazole; UK: United Kingdom; UTI: Urinary tract infection
}

\section{Acknowledgements}

We thank Professor Miguel Moreno and Dr. Christina Greko for helping to set up links with some collaborating laboratories.

\section{Funding}

This study was conducted with the financial support of CIISA and Fundação para a Ciência e a Tecnologia through Project UID/CVT/00276/2013. CM and AB hold Fundação para a Ciência e a Tecnologia PhD grants SFRH/BD/ 77886/2011 and SFRH/BD/113142/2015, respectively.

\section{Availability of data and material}

The information supporting the conclusions of this article is included within the article.

\section{Authors' contributions}

CP conceived the study and its coordination. CP and CM participated in the design of the study and contacted the collaborating laboratories included. All authors were responsible for data regarding the bacteria identification and susceptibility testing from the respective laboratory. CM and LTG conducted statistical analysis. CM wrote the initial draft and $K B, E M B, P D$, MAMD, RD, IL, DM, GO, VP, XR, JS, DT, GW, RGZ, SS, LG and CP made critical improvements for the final manuscript. All authors read and approved the final manuscript.

\section{Competing interests}

The authors declare that they have no competing interests.

\section{Consent for publication}

Not applicable.

\section{Ethics approval and consent to participate}

Not applicable.

\section{Author details}

${ }^{1}$ Faculdade de Medicina Veterinária, Centro de Investigação Interdisciplinar em Sanidade Animal (CIISA), Lisboa, Portugal. ${ }^{2}$ The National Veterinary Institute (SVA), Uppsala, Sweden. ${ }^{3}$ Laboratoire Vebiotel, Arcueil, France. ${ }^{4}$ Department of Infectious Diseases and Immunology, Utrecht University, Utrecht, The Netherlands. ${ }^{5}$ Department of Clinical Veterinary Sciences, Faculty of Medical and Veterinary Sciences, University of Bristol, Langford, UK. ${ }^{6} \mathrm{AML}-$ Medvet laboratory, Antwerp, Belgium. ${ }^{7}$ Department of Veterinary Disease Biology, Faculty of Health and Medical Sciences, University of Copenhagen, Frederiksberg, Denmark. ${ }^{8}$ Department of Clinical Sciences of Companion Animals, University of Utrecht, Utrecht, The Netherlands. ${ }^{9} \mathrm{Clinic}$ of Small Animal Medicine, LMU Munich, Munich, Germany. ${ }^{10}$ Hospital Clínico Veterinario Complutense, Universidad Complutense, Servicio de Microbiologia y Parasitologia, Madrid, Spain. ${ }^{11}$ Vetsuisse Faculty, University of Zurich, Clinic for Small Animal Internal Medicine, Zurich, Switzerland.

${ }^{12}$ Department of Clinical Studies, Companion Animal Clinic, Faculty of Health Sciences, School of Veterinary Medicine, Aristotle University of Thessaloniki, Thessaloniki, Greece. ${ }^{13}$ Institute of Microbiology, University of Veterinary Medicine, Vienna, Austria. ${ }^{14}$ Department of Veterinary Medical Science, University of Bologna, Bologna, Italy. ${ }^{15}$ Department of microbiology, Faculty of Veterinary Medicine, University of Belgrade, Belgrade, Serbia. ${ }^{16} \mathrm{Hospital}$ Clínic Veterinari, Universitat Autònoma de Barcelona, Bellaterra, Spain. ${ }^{17}$ Institute of Veterinary Bacteriology, Vetsuisse Faculty, University of Bern, Bern, Switzerland. ${ }^{18}$ School of Veterinary Science, University of Liverpool, Cheshire, UK. ${ }^{19}$ Faculty of Veterinary Medicine, University of Agronomical Sciences Veterinary Medicine, Lasi, Romania. ${ }^{20}$ Institute of Infection and Global Health, University of Liverpool, Liverpool, UK. ${ }^{21}$ Institute for Infectious Diseases and Zoonoses, LMU Munich, Munich, Germany. ${ }^{22}$ Institute of Veterinary Bacteriology, Vetsuisse Faculty, University of Zurich, Zurich, Switzerland. ${ }^{23}$ Department of Biomedical Sciences, Ross University School of Veterinary Medicine, Basseterre, St Kitts and Nevis.

Received: 19 September 2015 Accepted: 13 September 2016 Published online: 22 September 2016

\footnotetext{
References

1. Dowling PM. Antimicrobial therapy of urinary tract infections. Can Vet J. 1996;37:438-41.
} 
2. Thompson MF, Litster AL, Platell JL, Trott DJ. Canine bacterial urinary tract infections: New developments in old pathogens. Vet J. 2011;190:22-7.

3. Gerber B, Boretti FS, Kley S, Laluha P, Müller C, Sieber N, et al. Evaluation of clinical signs and causes of lower urinary tract disease in European cats. J Small Anim Pract. 2005;46:571-7.

4. Eggertsdóttir AV, Lund HS, Krontveit R, Sørum H. Bacteriuria in cats with feline lower urinary tract disease: a clinical study of 134 cases in Norway. J Feline Med Surg. 2007;9:458-65.

5. Saevik BK, Trangerud C, Ottesen N, Sørum H, Eggertsdóttir AV. Causes of lower urinary tract disease in Norwegian cats. J Feline Med Surg. 2011;13:410-7.

6. Mayer-Roenne B, Goldstein RE, Erb HN. Urinary tract infections in cats with hyperthyroidism, diabetes mellitus and chronic kidney disease. J Feline Med Surg. 2007;9:124-32.

7. Litster A, Moss SM, Honnery M, Rees B, Trott DJ. Prevalence of bacterial species in cats with clinical signs of lower urinary tract disease: Recognition of Staphylococcus felis as a possible feline urinary tract pathogen. Vet Microbiol. 2007:121:182-8.

8. Hall JL, Holmes MA, Baine SJ. Prevalence and antimicrobial resistance of canine urinary tract pathogens. Vet Rec. 2013;173:549.

9. Dorsch R, von Vopelius-Feldt C, Wolf G, Straubinger RK, Hartmann K. Feline urinary tract pathogens: prevalence of bacterial species and antimicrobial resistance over a 10-year period. Vet Rec. 2015;176:201.

10. Féria C, Correia J, Machado J, Vidal R, Gonçalves J. Urinary tract infection in dogs. Analysis of 419 urocultures carried out in Portugal. Adv Exp Med Biol. 2000:485:301-4

11. Normand EH, Gibson NR, Reid SWJ, Carmichael S, Taylor DJ. Antimicrobialresistance trends in bacteria isolates from companion-animal community practice in the UK. Prev Vet Med. 2000;46:267-78.

12. Cohn LA, Gary AT, Fales WH, Madsen RW. Trends in fluoroquinolone resistance of bacteria isolated from canine urinary tracts. J Vet Diagn Invest. 2003;15:338-43.

13. Pellerin JL, Bourdeau P, Sebbag H, Person JM. Epidemiosurveillance of antimicrobial compound resistance of Staphylococcus intermedius clinical isolates from canine pyodermas. Comp Immunol Microbiol Infect Dis. 1998;21:115-33.

14. European Medicine Agency. Reflection paper on the risk of antimicrobial resistance transfer from companion animals. http://www.ema.europa.eu/ema/ pages/includes/document/open_document.jsp?webContentld=WC500181642. Accessed 15 Sept 2015.

15. Pomba C, Endimiani A, Rossano A, Saial D, Couto N, Perreten V. First report of OXA-23-mediated carbapenem resistance in sequence type 2 multidrug-resistant Acinetobacter baumannii associated with urinary tract infection in a cat. Antimicrob Agents Chemother. 2014;58:1267-8.

16. Johnson JR, Clabots C. Sharing of virulent Escherichia coli clones among household members of a woman with acute cystitis. Clin Infect Dis. 2006;43:e101-8.

17. Guardabassi L, Schwarz S, Lloyd H. Pet animals as reservoirs of antimicrobial-resistant bacteria. J Antimicrob Chemother. 2004;54:321-32.

18. European Centre for Disease Prevention and Control. Antimicrobial resistance surveillance in Europe 2013. Annual Report of the European Antimicrobial Resistance Surveillance Network (EARS-Net). Stockholm: ECDC; 2014

19. Weese S, Blondeau JM, Boothe D, Breitschwerdt EB, Guardabassi L, Hillier A, et al. Antimicrobial use guidelines for treatment of urinary tract disease in dogs and cats: antimicrobial guidelines working group of the international society for companion animal infectious disease. Vet Med Int. 2011;2011:263768.

20. World Organization for Animal Health. OIE Terrestrial Animal Health Code Chapter 6.9. Responsible and Prudent Use of Antimicrobial Agents in Veterinary Medicine. http://www.oie.int/index.php?id=169\&L=0\&htmfile= chapitre_antibio_use.htm. Accessed 15 Sept 2015.

21. Clinical and Laboratory Standards Institute. Performance Standards for Antimicrobial Disk and Dilution Susceptibility Tests for Bacteria Isolated from Animals-Fourth Edition: Approved Standard VET01-A4. Wayne: CLSI; 2013.

22. Clinical and Laboratory Standarts Institute. Performance Standards for Antimicrobial Susceptibility Testing: Twenty-third Informational Supplement M100-S25. Wayne: CLSI; 2015

23. Clinical and Laboratory Standarts Institute. Performance Standards for Antimicrobial Disk and Dilution Susceptibility Tests for Bacteria Isolated from Animals: Second Information Supplement VET01-S2. Wayne: CLSI; 2013.

24. Societe Française de Microbiology. Antibiogramme veterinaire du comite de l'antibiogramme de la societe francaise de microbiologie. Paris: SFM; 2012.
25. Howe RA, Andrews JM, Party BW. BSAC standardized disc susceptibility testing method (version 11). J Antimicrob Chemother. 2012;67:2783-4.

26. Commissie Richtlijnen Gevoeligheidsbepalingen. Interpretatie van gevoeligheidsonderzoek en gevoeligheidscriteria voor antibacteriële middelen in Nederland. Ned Tijdschr Med Microbiol. 2000;8:79-81.

27. Magiorakos AP, Srinivasan A, Carey RB, Carmeli Y, Falagas ME, Giske CJ, et al. Multidrug-resistant, extensively drug-resistant and pandrug-resistant bacteria: an international expert proposal for interim standard definitions for acquired resistance. Clin Microbiol Infect. 2012;18:268-81.

28. Ruscher C, L bke-Becker A, Wleklinski CG, Soba A, Wieler LH, Walther B. Prevalence of Methicillin-resistant Staphylococcus pseudintermedius isolated from clinical samples of companion animals and equidaes. Vet Microbiol. 2008;136:197-201

29. Meunier D, Acar JF, Martel JL, Kroemer S, Valle M. A seven-year survey of susceptibility to marbofloxacin of pathogenic strains isolated from pets. Int Antimicrob Agents. 2004;24:592-8.

30. Kroemer S, Garch FE, Galland D, Petit JL, Woehrle F, Boulouis HJ. Antibiotic susceptibility of bacteria isolated from infections in cats and dogs throughout Europe (2002-2009). Comp Immunol Microbiol Infect Dis. 2014;37:97-108.

31. Ball KR, Rubin JE, Chirino-Trejo M, Dowling PM. Antimicrobial resistance and prevalence of canine uropathogens at the Western College of Veterinary Medicine Veterinary Teaching Hospital, 2002-2007. Can Vet J. 2008:49:985-90.

32. Windahl U, Holst BS, Nyman A, Grönlund U, Bengtsson B. Characterisation of bacterial growth and antimicrobial susceptibility patterns in canine urinary tract infections. BMC Vet Res. 2014;10:217.

33. Féria C, Ferreira E, Correia JD, Gonçalves J, Caniça M. Patterns and mechanisms of resistance to $\beta$-lactams and $\beta$-lactamase inhibitors in uropathogenic Escherichia coli isolated from dogs in Portugal. J Antimicrob Chemother. 2002:49:77-85.

34. Grobbel M, Lübke-Becker A, Alešík E, Schwarz S, Wallmann J, Werckenthin C, et al. Antimicrobial susceptibility of Escherichia coli from swine, horses, dogs and cats as determined in the BfT-GermVet monitoring program 20042006. Berl Munch Tierarztl Wochenschr. 2007;120:391-401.

35. Lanz R, Kuhnert P, Boerlin P. Antimicrobial resistance and resistance gene determinants in clinical Escherichia coli from different animal species in Switzerland. Vet Microbiol. 2003;91:73-84.

36. Criel D, Steenbergen J, Stalpaert M. Prevalence and antimicrobial susceptibility of canine uropathogens in Northern Belgium: a retrospective study (2010 to 2012). J Small Anim Pract. 2015;56:73.

37. Pedersen $K$, Pedersen $K$, Jensen $H$, Finster $K$, Jensen VF, Heuer OE. Occurrence of antimicrobial resistance in bacteria from diagnostic samples from dogs. J Antimicrob Chemother. 2007;60:775-81.

38. Hagman R, Greko C. Antimicrobial resistance in Escherichia coli isolated from bitches with pyometra and from urine samples from other dogs. Vet Rec. 2005;157:193-7.

39. World Health Organization. Critically important antimicrobials for human medicine. http://apps.who.int/iris/bitstream/10665/77376/1/9789241504485_ eng.pdf. Accessed 15 Sept 2015.

40. Swedres-Svarm. Consumption of antibiotics and occurrence of antibiotic resistance in Sweden. Solna/Uppsala: Public Health Agency of Sweden and National Veterinary Institute; 2014.

41. Guardabassi L, Prescott JF. Antimicrobial stewardship in small animal veterinary practice: From theory to practice. Vet Clin North Am Small Anim Pract. 2015:45:361-76.

42. European Committee on Antimicrobial Susceptibility Testing. EUCAST guidelines for detection of resistance mechanisms and specific resistances of clinical and/or epidemiological importance. Version 1.0. http://www.eucast.org/fileadmin/src/media/PDFs/EUCAST_files/ Resistance_mechanisms/EUCAST_detection_of_resistance_mechanisms_ v1.0_20131211.pdf. Accessed 15 Sept 2015.

43. Tramuta C, Robino P, Nucera D, Salvarani S, Banche G, Malabaila A, et al. Molecular characterization and antimicrobial resistance of faecal and urinary Escherichia coli isolated from dogs and humans in Italy. Vet Ital. 2014:50:23-30.

44. Oliveira M, Dias FR, Pomba C. Biofilm and fluoroquinolone resistance of canine Escherichia coli uropathogenic isolates. BMC Res Notes. 2014:7:499.

45. Grobbel M, Lübke-Becker A, Alešik E, Schwarz S, Wallmann J, Werckenthin C, et al. Antimicrobial susceptibility of Klebsiella spp. and Proteus spp. from various organ systems of horses, dogs and cats as determined in the BfT-GermVet monitoring program 2004-2006. Berl Munch Tierarztl Wochenschr. 2007;120:402-11. 
46. Pomba C, Couto N, Moodley A. Treatment of a lower urinary tract infection in a cat caused by a multi-drug methicillin-resistant Staphylococcus pseudintermedius and Enterococcus faecalis. J Feline Med Surg. 2010;12:802-6.

47. Wagner S, Gally DL, Argyle SA. Multidrug-resistant Escherichia coli from canine urinary tract infections tend to have commensal phylotypes, lower prevalence of virulence determinants and ampC-replicons. Vet Microbiol. 2014;169:171-8.

48. Veterinary Committee on Antimicrobial Susceptibility Testing (VetCAST). http:// www.eucast.org/ast_of_veterinary_pathogens (2015). Accessed 15 Sept 2015.

Submit your next manuscript to BioMed Central and we will help you at every step:

- We accept pre-submission inquiries

- Our selector tool helps you to find the most relevant journal

- We provide round the clock customer support

- Convenient online submission

- Thorough peer review

- Inclusion in PubMed and all major indexing services

- Maximum visibility for your research

Submit your manuscript at www.biomedcentral.com/submit 